\title{
Dissipative solitons in fiber lasers
}

\author{
S K Turitsyn, N N Rosanov, I A Yarutkina, A E Bednyakova, S V Fedorov, \\ O V Shtyrina, M P Fedoruk
}

\section{Contents}

1. Introduction. Optical solitons

000

2. Basic mathematical models

000

2.1 Maxwell-Bloch model; 2.2 Mathematical models of the basic elements of a fiber laser; 2.3 Vector model of the field evolution taking nonlinear optical rotation into account

3. Basic analytic solutions of the laser Haus-Ginzburg-Landau equation

3.1 Dissipative soliton solutions; 3.2 Automodel solutions; 3.3 Other analytic solutions

4. Poincaré map in describing laser systems

5. Simplified models describing the dynamics of laser pulses

6. Dissipative Maxwell-Bloch solitons

7. Dissipative self-induced transparency solitons

8. Examples of dissipative solitons in fiber lasers

8.1 Dissipative solitons in long fiber lasers; 8.2 Dissipative solitons in a SESAM mode-locked femtosecond fiber laser; 8.3 Dissipative solitons in a nonlinear optical-rotation mode-locked laser; 8.4 Raman dissipative solitons; 8.5 Adiabatic soliton laser

9. Conclusions and an outlook

Abstract. Dissipative solitons (also known as auto-solitons) are stable, nonlinear, time- or space-localized solitary waves that occur due to the balance between energy excitation and dissipation. We review the theory of dissipative solitons applied to fiber laser systems. The discussion context includes the classical Ginzburg-Landau and Maxwell-Bloch equations and their

S K Turitsyn Aston Institute of Photonic Technologies, Aston University, Aston Triangle, Birmingham, B4 7ET, United Kingdom; Novosibirsk State University,

ul. Pirogova 2, 630090 Novosibirsk, Russian Federation E-mail: s.k.turitsyn@aston.ac.uk

N N Rosanov, S V Fedorov Joint Stock Company

'Vavilov State Optical Institute',

Kadetskaya liniya V.O. 5, korp. 2, 199053 St. Petersburg, Russian Federation;

St. Petersburg National Research University of Information Technologies, Mechanics and Optics,

Kronverksky pr. 49, 197101 St. Petersburg, Russian Federation

E-mai:nnrosanov@mail.ru, sfedorov2006@bk.ru

I A Yarutkina, A E Bednyakova, O V Shtyrina, M P Fedoruk

Novosibirsk State University,

ul. Pirogova 2, 630090 Novosibirsk, Russian Federation;

Institute of Computational Technologies of the Siberian Branch

of the Russian Academy of Sciences,

prosp. Akademika Lavrent'eva 6, 630090 Novosibirsk,

Russian Federation

E-mail: mife@ict.nsc.ru

Received 23 November 2015

Uspekhi Fizicheskikh Nauk 186 (7) 713 - 742 (2016)

DOI: 10.3367/UFNr.2015.12.037674

Translated by M Sapozhnikov; edited by A M Semikhatov modifications allowing the description of laser-cavity-produced waves. Practical examples of laser systems generating dissipative solitons are discussed.

Keywords: dissipative solitons, optical solitons, fiber lasers, mode-locked lasers, generation of short pulses

\section{Introduction. Optical solitons}

The concept of solitons [1] - stable, localized, particle-like physical structures produced in the nonlinear interaction of distributed waves (physical fields) - is one of the fundamental unifying ideas of modern theoretical physics and mathematics [2-21]. The energy localization of a space-time distributed field under the action of nonlinearity is a quite general physical phenomenon appearing in different fields of physics. The theory of solitons has found applications in practical and applied problems in various fields of science such as hydrodynamics, plasma physics, nonlinear optics, molecular biology, field theory, and astrophysics. Dynamic solitons appear due to the balance between linear (for example, dispersion or diffraction) and nonlinear effects. More complex solitons exist, for example, topological solitons, which appear in systems with topologically nontrivial ground states. Examples of topological solitons include vortices, domain walls, optical $2 \pi$-pulses, and other structures (see details in [22-24]).

In the theory of integrable nonlinear equations, in particular, the inverse spectral transform theory, the soliton solutions of an integrable partial differential equation correspond to reflectionless potentials for the corresponding scattering problems $[3,4,12-14]$. In the mathematical 
literature, the term 'soliton' is often used only to refer to the solutions of integrable equations, whereas the term 'solitary nonlinear wave' is used for nonintegrable models. However, many characteristics of solitons, important in applications, are not related to the integrability of the corresponding mathematical models. In this review, we use the term soliton in a broad sense by considering nonintegrable nonlinear models that have solutions describing coherent localized structures. A stable (particle-like) behavior is a key characteristic in such a physical definition of solitons.

Two important classes of solitons, conservative and dissipative, can be distinguished. Conservative solitons appear in so-called transparent media, where losses are negligibly small, the energy supply to the system is absent, and localization is the result of a balance between the linear spread of a wave packet (for example, due to diffraction or dispersion) and nonlinear compression (for example, selffocusing). Optical dissipative solitons (autosolitons) are stable nonlinear light, but localized wave packets with the balanced energy supply and loss in a physical system. In the theory of dissipative solitons, it is often impossible to separate conservative and dissipative effects.

The history of optical solitons is closely connected with two basic models: the Ginzburg-Landau equation and the nonlinear Schrödinger equation (NSE). They are used to describe a variety of physical phenomena. The GinzburgLandau equation is widely used in physics to describe the dynamics of dissipative nonlinear systems in the presence of amplification, losses, and other effects depending on the specificity of a physical problem, for example, linear or nonlinear dispersion, saturating amplification, and (or) losses. This equation was first proposed by Ginzburg and Landau in the context of phase transitions in the theory of superconductivity [25]. Since then, the Ginzburg-Landau equation has been used for describing various physical phenomena, including convection [26, 27], the theory of mode-locked lasers [28], general models in nonlinear optics [29], and many other physical problems (see, e.g., [9, 10, 30] and the references therein).

The NSE predecessor appeared in Bogoliubov's microscopic theory of superfluidity (the model of a weakly interacting Bose gas) [31] describing the spectrum of a condensate. We note that the possibility of instability (widely known as the modulation instability) was also mentioned in this classic work in 1947. In the 1960s, the classic NSE was applied for studying the atomic Bose gas and became known in this field as the Gross-Pitaevskii equation $[32,33]$. Somewhat later, the NSE was used to analyze highpower laser beams [34-37] and to describe the hydrodynamic wave instability [38]. The NSE is widely used in many other important physical applications [39]. In 1972, Zakharov and Shabat demonstrated the NSE integrability, which initiated numerous investigations of the mathematical properties of this model [40]. Mathematically, the NSE can be considered as the conservative limit of the generalized complex Ginzburg-Landau equation. The NSE is one of the most important nonlinear mathematical models in optics and is applied in optical signal processing, data transmission, pulse compression, optical signal shaping, frequency converters, and various nonlinear optical devices. Many of these applications are based on the existence of a stable NSE solution in the form of a fundamental soliton. Because the optical soliton is a stable pulse and can be used as an elementary information bit, it plays an important role in nonlinear concepts of data communication and digital data storage and processing.

In this review, we focus our attention on dissipative solitons. First, we consider dissipative solitons in the context of the Ginzburg-Landau and Maxwell-Bloch equations. We then discuss methods for describing dissipative solitons in a laser cavity. And finally, we present examples of practical laser systems in which dissipative solitons appear.

\section{Basic mathematical models}

\subsection{Maxwell-Bloch model}

Basic models for describing a fiber laser are constructed using Maxwell's equations of the electrodynamics of continuous media supplemented with corresponding matter equations. We assume that all materials forming a laser medium are nonmagnetic, with the magnetic permeability equal to unity. Of practical interest is the single transverse mode regime, and the transverse structure of the field can therefore be assumed to be fixed. We also assume for simplicity that the polarization of radiation is fixed; then Maxwell's equations reduce to scalar one-dimensional equations

$$
\frac{1}{c} \frac{\partial \tilde{D}}{\partial T}-\frac{\partial \tilde{H}}{\partial z}=0, \quad \frac{1}{c} \frac{\partial \tilde{H}}{\partial T}-\frac{\partial \tilde{E}}{\partial z}=0
$$

where $z$ is the longitudinal coordinate (along the laser axis), $T$ is time, $c$ is the speed of light in a vacuum, and $\tilde{E}, \tilde{H}$, and $\tilde{D}$ are the electric and magnetic strength and electric induction components. Further simplifications are achieved for the unidirectional propagation of quasi-monochromatic radiation (the slowly varying envelope approximation) in a medium with a fast (inertialess) response. For such radiation, a slowly varying envelope $A$ (compared to the mean optical period $\left.2 \pi / \omega_{0}\right)$ can be introduced by the relation

$$
\tilde{E}(z, T)=\operatorname{Re}\left[A(z, T) \exp \left(\mathrm{i} k_{0} z-\mathrm{i} \omega_{0} T\right)\right],
$$

where $k_{0}$ is the wave number for the carrier frequency $\omega_{0}$. Then the propagation of a pulse through a fiber with the linear absorption coefficient $\Gamma$ and the Kerr nonlinearity coefficient $\gamma$ of the refractive index is described in the coordinate system comoving with the pulse with a group velocity $v_{\mathrm{g}}\left(t=T-z / v_{\mathrm{g}}\right)$ by the standard generalized NSE [41]

$$
\frac{\partial A}{\partial z}=-\mathrm{i} \frac{\beta_{2}}{2} \frac{\partial^{2} A}{\partial t^{2}}+\frac{\beta_{3}}{6} \frac{\partial^{3} A}{\partial t^{3}}+\mathrm{i} \gamma|A|^{2} A-\Gamma A,
$$

where $\beta_{2}$ and $\beta_{3}$ are the second- and third-order dispersion coefficients (higher-order dispersion is neglected). All the parameters in this equation are effective characteristics integrated over the transverse dependence of the field for a particular mode of an optical fiber [41].

In the case of the resonance nonlinearity of a medium realized under laser amplification or saturable absorption, the form of matter equations requires specifying the medium model. The medium polarizability $\tilde{P}$ is introduced by the relation $\tilde{D}=\tilde{E}+4 \pi \tilde{P}$ and is defined as the specific dipole moment of the medium. For fiber amplifiers and lasers, where amplification is provided by pumping rare-earth impurity ions in a matrix, the resonance nonlinearity is typical. Because the relaxation time of operating levels is quite long, the resonance response cannot be considered to be instant 


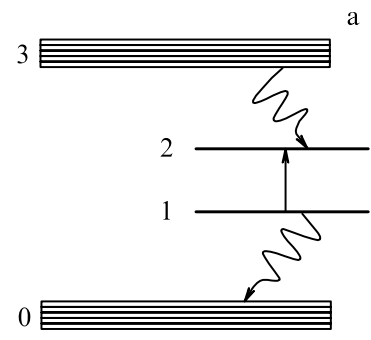

Figure 1. Level diagram.

(inertialess), while the finite spectral width of amplification and absorption bands automatically leads to quadratic and higher-order dispersion. The limit cases are the slow [28, 42, 43] and fast [44-48] relaxations. In this review, we consider only two limit cases: comparatively long laser pulses, for which the slowly varying amplitude approximation can be used, and extremely short laser pulses with the spectral width comparable to the central frequency. The active elements of fiber lasers are silica fibers doped with rare-earth ions $\mathrm{Nd}, \mathrm{Yb}$, $\mathrm{Er}, \mathrm{Ho}$, and Tm. Lasing occurs in the three- or four-level arrangement (Fig. 1a). Pumping excites a group 3 of high levels of active centers, from which fast relaxation occurs to the high operating level 2 , and hence the model reduces to the effective two-level arrangement (Fig. 1b) [49]. As a result, the resonance interaction of radiation with active centers is described by equations for the density matrix (the superscript (a) refers to an active center),

$$
\begin{aligned}
& \frac{\partial}{\partial T} \rho_{21}^{(\mathrm{a})}=-\left(\mathrm{i} \omega_{21}^{(\mathrm{a})}+\frac{1}{T_{2}^{(\mathrm{a})}}\right) \rho_{21}^{(\mathrm{a})}-\mathrm{i} \frac{\mu_{\mathrm{a}} \tilde{E}}{\hbar}\left(\rho_{22}^{(\mathrm{a})}-\rho_{11}^{(\mathrm{a})}\right), \\
& \frac{\partial}{\partial T} \rho_{22}^{(\mathrm{a})}=-\frac{1}{T_{1}^{(\mathrm{a})}} \rho_{22}^{(\mathrm{a})}-\mathrm{i} \frac{\mu_{\mathrm{a}} \tilde{E}}{\hbar}\left(\rho_{21}^{(\mathrm{a})}-\rho_{12}^{(\mathrm{a})}\right)+p \\
& \frac{\partial}{\partial T} \rho_{11}^{(\mathrm{a})}=\frac{1}{T_{1}^{(\mathrm{a})}} \rho_{22}^{(\mathrm{a})}-\frac{1}{T_{11}^{(\mathrm{a})}} \rho_{11}^{(\mathrm{a})}+\mathrm{i} \frac{\mu_{\mathrm{a}} \tilde{E}}{\hbar}\left(\rho_{21}^{(\mathrm{a})}-\rho_{12}^{(\mathrm{a})}\right) .
\end{aligned}
$$

Here, $\rho_{11}$ and $\rho_{22}$ are populations of the upper 1 and lower 2 operating levels with lifetimes $T_{1}^{(\mathrm{a})}$ and $T_{11}^{(\mathrm{a})}$, nondiagonal elements $\rho_{12}=\rho_{21}^{*}$ determine the dipole moment of the medium (see below), $\omega_{21}$ is the transition frequency, $\mu$ is the dipole transition matrix element, $p$ is the upper level pump rate, $T_{2}^{(\mathrm{a})}$ is the dipole moment relaxation time, and $\hbar$ is the Planck constant. In the case of homogeneous broadening (all active centers with the concentration $n_{a}$ are identical), the polarization is $\tilde{P}^{(\mathrm{a})}=n_{\mathrm{a}} \mu_{\mathrm{a}} \rho_{12}^{(\mathrm{a})}+$ c.c. Inhomogeneous broadening is described by the statistical scatter of parameters of the centers (first of all, the transition frequency). A two-level saturable absorber (the superscript (p)), for which pumping is absent and the lower level 1 can be assumed to be the ground level $\left(T_{11}^{(\mathrm{p})}=\infty\right)$, is described similarly:

$$
\begin{aligned}
& \frac{\partial}{\partial T} \rho_{21}^{(\mathrm{p})}=-\left(\mathrm{i} \omega_{21}^{(\mathrm{p})}+\frac{1}{T_{2}^{(\mathrm{p})}}\right) \rho_{21}^{(\mathrm{p})}-\mathrm{i} \frac{\mu_{\mathrm{p}} \tilde{E}}{\hbar}\left(\rho_{22}^{(\mathrm{p})}-\rho_{11}^{(\mathrm{p})}\right) \\
& \frac{\partial}{\partial T} \rho_{22}^{(\mathrm{p})}=-\frac{1}{T_{1}^{(\mathrm{p})}} \rho_{22}^{(\mathrm{p})}-\mathrm{i} \frac{\mu_{\mathrm{p}} \tilde{E}}{\hbar}\left(\rho_{21}^{(\mathrm{p})}-\rho_{12}^{(\mathrm{p})}\right) \\
& \frac{\partial}{\partial T} \rho_{11}^{(\mathrm{p})}=\frac{1}{T_{1}^{(\mathrm{p})}} \rho_{22}^{(\mathrm{p})}+\mathrm{i} \frac{\mu_{\mathrm{p}} \tilde{E}}{\hbar}\left(\rho_{21}^{(\mathrm{p})}-\rho_{12}^{(\mathrm{p})}\right)
\end{aligned}
$$

In this case, the total population of two operating levels is conserved: $\rho_{11}^{(\mathrm{p})}+\rho_{22}^{(\mathrm{p})}=1$. Taking the low concentration of resonance centers into account, their contribution to medium polarization can be assumed to be additive, and hence for homogeneous broadening we have

$$
\begin{aligned}
& \tilde{P}=\tilde{P}^{(\mathrm{m})}+\tilde{P}^{(\mathrm{a}, \mathrm{p})}, \\
& \tilde{P}^{(\mathrm{a}, \mathrm{p})}=n_{\mathrm{p}} \mu_{\mathrm{p}} \rho_{12}^{(\mathrm{p})}+n_{\mathrm{a}} \mu_{\mathrm{a}} \rho_{12}^{(\mathrm{a})}+\text { c.c. }
\end{aligned}
$$

The term $\tilde{P}^{(\mathrm{m})}$ describes the nonresonant part of the matrix polarization with the linear component $\left[\left(\hat{\varepsilon}_{0}-1\right) /(4 \pi)\right] \tilde{E}$ and the nonlinear component including the Kerr nonlinearity factor $\gamma$. Here, $\hat{\varepsilon}_{0}$ is the permittivity of the matrix nonresonantly interacting with radiation, its operator form taking the frequency dispersion of the matrix into account.

The system of equations (1), (4)-(6) is sufficient for simulating the propagation of extremely short optical pulses in a fiber, nonresonantly and resonantly interacting with the medium. Simplifications are achieved in the regime of unidirectional quasi-monochromatic radiation. Similarly to (2), we can then introduce the slowly varying polarization

$$
\tilde{P}(z, T)=\operatorname{Re}\left[\bar{P}(z, T) \exp \left(\mathrm{i} k_{0} z-\mathrm{i} \omega_{0} T\right)\right] .
$$

In this case, quasi-optical equation (3) is replaced by

$$
\frac{\partial A}{\partial z}=-\mathrm{i} \frac{\beta_{2}}{2} \frac{\partial^{2} A}{\partial t^{2}}+\frac{\beta_{3}}{6} \frac{\partial^{3} A}{\partial t^{3}}+\mathrm{i} \gamma|A|^{2} A-\Gamma A+\hat{G} A,
$$

where $\hat{G} A=2 \pi \mathrm{i}\left[k_{0} / \varepsilon_{0}\left(\omega_{0}\right)\right] \bar{P}^{(\mathrm{a}, \mathrm{p})}$. The real part of this expression for the zero phase of the amplitude $A$ is proportional to the gain (for active parts of the fiber) or saturable absorption, while the imaginary part is proportional to the resonance component of the nonlinear refractive index Amplification and resonance absorption are proportional to the difference between populations of two operating levels. Therefore, it is convenient to pass from Eqns (4) and (5) for the density matrix to Bloch equations for macroscopic quantities, resonance polarization (7) and the population difference $N^{(\mathrm{a}, \mathrm{p})}=n_{\mathrm{a}, \mathrm{p}}\left[\rho_{22}^{(\mathrm{a}, \mathrm{p})}-\rho_{11}^{(\mathrm{a}, \mathrm{p})}\right]$. In the resonance approximation, ignoring rapidly oscillating quantities, for a saturable absorber, instead of (5), we obtain

$$
\begin{aligned}
& \frac{\partial \bar{P}^{(\mathrm{p})}}{\partial T}=-\left(\mathrm{i} \delta \omega^{(\mathrm{p})}+\frac{1}{T_{2}^{(\mathrm{p})}}\right) \bar{P}^{(\mathrm{p})}-\mathrm{i} \frac{\mu_{\mathrm{p}}^{2} A}{\hbar} N^{(\mathrm{p})}, \\
& \frac{\partial N^{(\mathrm{p})}}{\partial T}=-\frac{1}{T_{1}^{(\mathrm{p})}}\left(N^{(\mathrm{p})}-N_{0}^{(\mathrm{p})}\right)-\frac{b^{(\mathrm{p})}}{\hbar} \operatorname{Im}\left(A \bar{P}^{(\mathrm{p}) *}\right),
\end{aligned}
$$

where $\delta \omega^{(\mathrm{p})}=\omega_{21}^{(\mathrm{p})}-\omega_{0}$ is the working transition detuning from the carrier frequency, $N_{0}^{(\mathrm{p})}$ is the equilibrium population difference in the absence of the field (in the case under study, $N_{0}^{(\mathrm{p})}=-n$, i.e., all the centers in the absence of radiation are found at the lower level), and $b^{(\mathrm{p})}=1$ (the value of this parameter is determined by whether the lower level is the ground level). In the comoving coordinate system, the derivative $\partial / \partial T$ in the left-hand sides of (9) is replaced by $\partial / \partial t$

For amplification in the four-level arrangement, assuming the rapid decay of the lower working level $\left(T_{11}^{(\mathrm{a})} \ll T_{1}^{(\mathrm{a})}\right.$, $\left.\rho_{11}^{(\mathrm{a})} \ll \rho_{22}^{(\mathrm{a})}\right)$, we obtain the same equations with the replacement of superscripts $(\mathrm{p}) \rightarrow(\mathrm{a}), \quad N_{0}^{(\mathrm{a})}=n p T_{1}^{(\mathrm{a})}$, and $b^{(a)}=1 / 2$. Bloch equations (9) are required for the description of the coherent self-mode-locking regime (the pulse duration is considerably shorter than the relaxation times 
$\left.T_{2}^{(\mathrm{a}, \mathrm{p})}\right)$ in the slowly varying envelope approximation [50]. In the most widespread incoherent self-mode-locking regime (relaxation times $T_{2}^{(\mathrm{a}, \mathrm{p})}$ are small compared to the pulse duration), polarization rapidly sets in and, as follows from the first equation in (9),

$$
\bar{P}^{(\mathrm{a}, \mathrm{p})}=-\mathrm{i} \frac{\mu_{\mathrm{a}, \mathrm{p}}^{2} A}{\hbar} \frac{N^{(\mathrm{a}, \mathrm{p})}}{\mathrm{i} \delta \omega^{(\mathrm{a}, \mathrm{p})}+1 / T_{2}^{(\mathrm{a}, \mathrm{p})}} .
$$

Equations (9) are then reduced to kinetic equations for the population differences,

$$
T_{1}^{(\mathrm{a}, \mathrm{p})} \frac{\partial N^{(\mathrm{a}, \mathrm{p})}}{\partial t}=N_{0}^{(\mathrm{a}, \mathrm{p})}-\left[1+\frac{|A|^{2}}{I_{\mathrm{s}}^{(\mathrm{a}, \mathrm{p})}}\right] N^{(\mathrm{a}, \mathrm{p})},
$$

which supplement and close Eqns (8) and (10). Here, the saturation intensity

$$
I_{\mathrm{s}}^{(\mathrm{a}, \mathrm{p})}=\frac{\hbar^{2}}{b^{(\mathrm{a}, \mathrm{p})} \mu_{\mathrm{a}, \mathrm{p}}^{2}} \frac{T_{2}^{(\mathrm{a}, \mathrm{p})}}{T_{1}^{(\mathrm{a}, \mathrm{p})}}\left[\left(\delta \omega^{(\mathrm{a}, \mathrm{p})}\right)^{2}+\frac{1}{\left(T_{1}^{(\mathrm{a}, \mathrm{p})}\right)^{2}}\right]
$$

is introduced.

Finally, in the quasi-continuous regime with $\partial N^{(\mathrm{a}, \mathrm{p})} / \partial t=0$ ('fast' amplifying and absorbing media), population differences change adiabatically with the radiation intensity $I=|A|^{2}$ :

$$
N^{(\mathrm{a}, \mathrm{p})}=\frac{N_{0}^{(\mathrm{a}, \mathrm{p})}}{1+I / I_{\mathrm{s}}^{(\mathrm{a}, \mathrm{p})}} .
$$

In this case, the field dynamics are described by the unified equation

$$
\begin{aligned}
\frac{\partial A}{\partial z} & =-\mathrm{i} \frac{\beta_{2}}{2} \frac{\partial^{2} A}{\partial t^{2}}+\frac{\beta_{3}}{6} \frac{\partial^{3} A}{\partial t^{3}}+\mathrm{i} \gamma|A|^{2} A-\Gamma A+\frac{2 \pi k_{0}}{\hbar \varepsilon_{0}\left(\omega_{0}\right)} \\
& \times \sum_{\mathrm{m}=\mathrm{a}, \mathrm{p}} \frac{\mu_{\mathrm{m}}^{2}}{\mathrm{i} \delta \omega^{(\mathrm{m})}+1 / T_{2}^{(\mathrm{m})}} \frac{N_{0}^{(\mathrm{m})}}{1+I / I_{\mathrm{s}}^{(\mathrm{m})}} A .
\end{aligned}
$$

We note that the equilibrium values of the population difference for the amplifier and absorber have opposite signs: $N_{0}^{(\mathrm{a})}>0$ and $N_{0}^{(\mathrm{p})}<0$. With the frequency selectivity of amplification and losses in the approximation quadratic in the frequency deviation taken into account, the coefficient $\beta_{2}$ becomes complex.

Although the above equations are obtained for a 'singlepass' fiber, they can be generalized for the dynamics of a fiber laser if the relative changes in the radiation and amplification (absorption) amplitudes per pass are small and the pulse duration $\tau_{p}$ and relaxation times of the medium are shorter than the round-trip time $T_{\mathrm{R}}$ in the cavity [28, 43]. Then (small) lumped losses on laser mirrors can be replaced by losses distributed over the cavity length $L: \Gamma=\left(1-R_{m}\right) / L$, where $R_{m}$ is the product of the amplitude reflection coefficients of the ring cavity mirrors. Another consequence of the assumption about the small change in the radiation amplitude per pass is that the resonance amplification and absorption of the medium only weakly change per pass under the action of a pulse wit the duration $\tau_{p}$ much shorter than the round-trip time $T_{\mathrm{R}}$ of light in the cavity. As a result, amplification and absorption can be averaged over time $T_{\mathrm{R}}$ : $N^{(\mathrm{a}, \mathrm{p})} \rightarrow\left\langle N^{(\mathrm{a}, \mathrm{p})}\right\rangle=T_{\mathrm{R}}^{-1} \int_{0}^{T_{\mathrm{R}}} N^{(\mathrm{a}, \mathrm{p})}(z, t) \mathrm{d} t$. By introducing the saturation energy density $W_{\mathrm{s}}^{(\mathrm{a}, \mathrm{p})}=I_{\mathrm{s}}^{(\mathrm{a}, \mathrm{p})} T_{\mathrm{R}}$, we use (11) to obtain

$$
\left\langle N^{(\mathrm{a}, \mathrm{p})}\right\rangle=\frac{N_{0}^{(\mathrm{a}, \mathrm{p})}}{1+1 / W_{\mathrm{s}}^{(\mathrm{a}, \mathrm{p})} \int_{0}^{T_{\mathrm{R}}}\left(|A(z, t)|^{2}\right) \mathrm{d} t} .
$$

Accordingly, the field dynamic equation takes the form

$$
\begin{aligned}
\frac{\partial A}{\partial z} & =-\mathrm{i} \frac{\beta_{2}}{2} \frac{\partial^{2} A}{\partial t^{2}}+\frac{\beta_{3}}{6} \frac{\partial^{3} A}{\partial t^{3}}+\mathrm{i} \gamma|A|^{2} A-\Gamma A \\
& +\frac{2 \pi k_{0}}{\hbar \varepsilon_{0}\left(\omega_{0}\right)} \sum_{\mathrm{m}=\mathrm{a}, \mathrm{p}} \frac{\mu_{\mathrm{m}}^{2}}{\mathrm{i} \delta \omega^{(\mathrm{m})}+1 / T_{2}^{(\mathrm{m})}} \\
& \times \frac{N_{0}^{(\mathrm{m})}}{1+1 / W_{\mathrm{s}}^{(\mathrm{m})} \int_{0}^{T_{\mathrm{R}}}\left(|A(z, t)|^{2}\right) \mathrm{d} t} A .
\end{aligned}
$$

In addition to this case of a "slow amplifier and absorber," also of interest is the mixed case of the "slow amplifier and fast absorber," combining (14) and (16):

$$
\begin{aligned}
\frac{\partial A}{\partial z} & =-\mathrm{i} \frac{\beta_{2}}{2} \frac{\partial^{2} A}{\partial t^{2}}+\frac{\beta_{3}}{6} \frac{\partial^{3} A}{\partial t^{3}}+\mathrm{i} \gamma|A|^{2} A-\Gamma A+\frac{2 \pi k_{0}}{\hbar \varepsilon_{0}\left(\omega_{0}\right)} \\
& \times\left[\frac{\mu_{\mathrm{a}}^{2}}{\mathrm{i} \delta \omega^{(\mathrm{a})}+1 / T_{2}^{(\mathrm{a})}} \frac{N_{0}^{(\mathrm{a})}}{1+1 / W_{\mathrm{s}}^{(\mathrm{a})} \int_{0}^{T_{\mathrm{R}}}\left(|A(z, t)|^{2}\right) \mathrm{d} t}\right. \\
& \left.+\frac{\mu_{\mathrm{p}}^{2}}{\mathrm{i} \delta \omega^{(\mathrm{p})}+1 / T_{2}^{(\mathrm{p})}} \frac{N_{0}^{(\mathrm{p})}}{1+I / I_{\mathrm{s}}^{(\mathrm{p})}}\right] A .
\end{aligned}
$$

In [51], the effective two-level model for an erbium amplifying medium was combined with the standard equation describing the propagation of light in an optical fiber for simulating an ytterbium fiber laser with strongly nonlinear intracavity radiation dynamics. An interesting and so far little-studied regime is the case where the round-trip time of a pulse in the laser cavity coincides with characteristic times in the active or passive medium. This can in principle be realized in fiber lasers with a long cavity [52-54] and can lead to new interesting lasing regimes.

\subsection{Mathematical models}

\section{of the basic elements of a fiber laser}

A passively mode-locked pulsed fiber laser can be simulated either using averaged models describing changes in the optical field after the round trip of radiation in the cavity and evolution from one transition to another or by applying the approach where each of the elements of the system is described by an individual model, and discrete elements, such as a saturable absorber, a coupler, or filters, should be considered separately from the evolution in the fiber. In Section 2.1, we described an ab initio derivation of equations describing amplification and a saturable absorber. For a practical analysis of laser systems, when not all the fundamental parameters of the laser system can be known, simpler heuristic models are often used with unknown parameters are determined from experiments. Below, we present the simplest mathematical models used for the practical description of the propagation of an optical pulse through the basic elements of a fiber laser.

Active and passive fibers. The propagation of a pulse through a laser fiber is described by generalized nonlinear Schrödinger equation (3)

The evolution of radiation in the active fiber is described by generalized Schrödinger equation (3), with the amplification and spectral filtration by the input field 
$A(t, z=0)=A_{\text {in }}(t)$ (which is determined by the previous round-trip transition in the cavity, except for the first roundtrip transition, when the initial field distribution is used).

The simulation of pulse propagation in the active fiber of a mode-locked laser should take the amplification saturation and filtering into account. The amplification saturation and filtering effects in the operator $\hat{G}$ are usually described in the frequency region using the Lorentzian profile

$$
\hat{G}(\omega)=\frac{1}{1+E / E_{\mathrm{sat} \mathrm{G}}} \frac{G_{0}}{1+\left[\left(\omega-\omega_{0}\right) / \Omega_{\mathrm{G}}\right]^{2}},
$$

where $\omega_{0}=2 \pi c / \lambda_{0}$ is the carrier frequency, $\Omega_{\mathrm{G}}=\left(2 \pi c / \lambda_{0}^{2}\right) \Lambda_{\mathrm{G}}$ is the filtration frequency, $c$ is the speed of light, $\Lambda_{\mathrm{G}}$ is the gain bandwidth, and $\lambda_{0}$ is the carrier wavelength of the laser. If the spectral filtration is mainly determined by the finite width of the gain band, then $\Omega_{\mathrm{G}}=1 / T_{2}^{(\mathrm{a})}$. The small-signal gain at the profile center is related to the microparameters presented in Section 2.1 as $G_{0}=\left(2 \pi k_{0} /\left(\hbar \varepsilon_{0}\right)\right) \mu_{\mathrm{a}}^{2} N_{0}^{(\mathrm{a})} T_{2}^{(\mathrm{a})}$. The amplification saturation occurs with increasing the pulse energy $E=\int_{0}^{T_{\mathrm{R}}}|A|^{2} \mathrm{~d} t$, the saturation energy being $E_{\mathrm{sat} \mathrm{G}}=$ $P_{\text {sat G }} T_{\mathrm{R}}$. Here, $P_{\text {sat G }}$ is the amplification saturation power. The first and second factors in (18) respectively correspond to amplification saturation and filtration.

A saturable absorber. The standard method for generating picosecond and femtosecond laser pulses is mode-locking. Passively mode-locked pulses are generated with the help of intracavity nonlinear elements, the pulse propagated through such elements becoming narrower. As a rule, such nonlinear elements are saturable absorbers (SAs). Passive mode-locking with the use of a saturable absorber is the simplest modelocking method, allowing the generation of narrower pulses.

A saturable absorber can operate in both light reflection and transmission regimes. In the reflection regime, for example, in semiconductor mirror saturable absorbers, the simplified transfer function describing the relation between input and output powers under the action of a saturable absorber has the form $T_{\text {refl }}(t)=1-q\left(t, P_{\text {in }}(t)\right)$ ( $w$ here $\left.P_{\text {in }}(t)=\left|A_{\text {in }}(t)\right|^{2}\right)$. At the same time, for a storable absorber based on in light transmission, for example, in carbon nanotubes, the transfer function has the form $T_{\text {trans }}(t)=$ $q\left(t, P_{\text {in }}(t)\right)$. The function $q$ can be found in both cases from the ordinary differential equation

$$
\frac{\mathrm{d} q}{\mathrm{~d} t}=-\frac{q-q_{0}}{T_{1}^{(\mathrm{p})}}-\frac{q|A(t)|^{2}}{T_{1}^{(\mathrm{p})} P_{\text {sat }}},
$$

where $q_{0}$ is the modulation depth, $P_{\text {sat }}$ is the absorption saturation power, and $T_{1}^{(\mathrm{p})}$ is the recovery time of the saturable absorber. We note that in the thin-layer approximation (small absorption losses), $q$ is proportional to the population difference $N^{(\mathrm{p})}$ of the operating levels introduced above: $q_{0}=\left(2 \pi k_{0} /\left(\hbar \varepsilon_{0}\right)\right) \mu_{\mathrm{p}}^{2}\left|N_{0}^{(\mathrm{p})}\right| T_{2}^{(\mathrm{p})} l$ and $q=\left(2 \pi k_{0} /\left(\hbar \varepsilon_{0}\right)\right) \mu_{\mathrm{p}}^{2}\left|N^{(\mathrm{p})}\right| T_{2}^{(\mathrm{p})} l$.

When the condition $\mathrm{d} q(t) / \mathrm{d} t=0$ is satisfied in the case of instant saturation, we obtain the simplified model

$$
q(t)=\frac{q_{0}}{1+\left|A_{\text {in }}(t)\right|^{2} / P_{\text {sat }}}
$$

We nte that the formal replacement of the point-wise action of elements by the effective distributed model may not coincide with the ab initio description presented in Section 2.1.

Coupler. The output field of a cavity propagating through a coupler has the form $P_{\text {out }}=R_{\text {out }} P_{\text {in }}$, where $P_{\text {in }}=\left|A_{\text {in }}\right|^{2}$ is the input field power of the coupler, $P_{\text {out }}=\left|A_{\text {out }}\right|^{2}$ is the total output power of the cavity, and $R_{\text {out }}$ are output power losses of the cavity. In this case, $P_{\text {cavity }}=\left(1-R_{\text {out }}\right) P_{\text {in }}$, where $P_{\text {cavity }}=\left|A_{\text {cavity }}\right|^{2}$ is the part of optical power remaining in the cavity after the propagation of light through the coupler.

\subsection{Vector model of the field evolution taking nonlinear optical rotation into account}

Besides a material saturable absorber, nonlinear physical effects can be ised to achieve mode-locking in a fiber laser. For example, a the popular method of passive mode-locking is based on nonlinear optical rotation of radiation propagating through a fiber when orthogonally polarized components of a pulse propagate through optical fibers and cause efficient absorption saturation $[55,56]$.

The substantial simplification made above in the formulation of the Ginzburg-Landau equation assumes that the incident beam polarization is preserved during its propagation in an optical fiber. Because this is not the case in fiber lasers mode-locked based on nonlinear optical rotation, the propagation of pulses in such lasers is simulated using the system of coupled Ginzburg-Landau equations (see, e.g., [57]). We consider the interaction of two orthogonally polarized components $A_{+}$and $A_{-}$of the optical field.

After propagation through a polarizer, the elliptically polarized components $A_{+}$and $A_{-}$of the slowly varying amplitude take the form

$$
\begin{aligned}
& A_{+}=A_{1} \cos \left(\chi-\frac{\pi}{4}\right) \exp (\mathrm{i} \psi), \\
& A_{-}=A_{1} \cos \left(\chi+\frac{\pi}{4}\right) \exp (-\mathrm{i} \psi),
\end{aligned}
$$

where $A_{1}$ is the input field, and $\chi$ and $\psi$ are the angles of rotation of two polarization plates (quarter-wave and halfwave, respectively), with $0<\chi<\pi / 4$.

To describe the evolution of the polarization vector in this case directly, it is necessary to use the system of coupled Ginzburg-Landau equations for the electromagnetic field amplitude $A(z, t)$ [57]

$$
\begin{aligned}
& \frac{\partial A_{+}}{\partial z}=\frac{\mathrm{i} \beta_{2}}{2} \frac{\partial^{2} A_{+}}{\partial t^{2}}+G A_{+}+\frac{\mathrm{i} \gamma}{3}\left(\left|A_{+}\right|^{2}-\left|A_{-}\right|^{2}\right) A_{+}-\mathrm{i} \gamma|A|^{2} A_{+}, \\
& \frac{\partial A_{-}}{\partial z}=\frac{\mathrm{i} \beta_{2}}{2} \frac{\partial^{2} A_{-}}{\partial t^{2}}+G A_{-}-\frac{\mathrm{i} \gamma}{3}\left(\left|A_{+}\right|^{2}-\left|A_{-}\right|^{2}\right) A_{-}-\mathrm{i} \gamma|A|^{2} A_{-} .
\end{aligned}
$$

Here, $|A|^{2}=\left|A_{+}\right|^{2}+\left|A_{-}\right|^{2}$ and $G$ is the signal gain. The components $A_{+}$and $A_{-}$of the optical field (the right- and left-hand circular polarizations of the electric field) propagating through a fiber can be represented in terms of linear polarizations as

$$
A_{1}=\frac{A_{+}+A_{-}}{\sqrt{2}}, \quad A_{2}=\frac{A_{+}-A_{-}}{\sqrt{2}} .
$$

The field component $A_{2}$ escapes from the cavity, while $A_{1}$ returns to the cavity and is polarized during its propagation in the cavity in accordance with system of equations (21). Here, components $A_{1}$ and $A_{2}$ are two linearly polarized components.

System of equations (22) describes the propagation of light in the fiber part of the cavity. By changing the angles of rotation of plates, the polarization of light (in particular, the 
ellipticity and the angle of the polarization ellipse axis), can be controlled at the fiber input. The effective parameters of such a saturable element are described in [58, 59].

\section{Basic analytic solutions of the laser Haus-Ginzburg-Landau equation}

At present, the complex Ginzburg-Landau equation is one of the best-known nonlinear models describing nonlinear effects in physics, such as nonlinear waves, second-order phase transitions, superconductivity, superfluidity, the Bose-Einstein condensate, liquid crystals, and numerous other phenomena [60]. The equation is a first-order approximation in the limit of a slowly varying amplitude for the electromagnetic field envelope characterizing the dynamics of nonlinear systems in the nonequilibrium state in the presence of losses (amplification). In optics, the Haus-Ginzburg-Landau equation is used to desctribe the propagation of optical pulses, in particular, to simulate mode-locked fiber lasers representing complex physical systems with linear and nonlinear dissipative elements $[9,42,59,61-64]$.

\subsection{Dissipative soliton solutions}

We consider the cubic Haus-Ginzburg-Landau equation, which is widely used for simulating passively mode-locked lasers [42]:

$$
\begin{aligned}
\frac{\partial A}{\partial z} & =-\frac{\mathrm{i} \beta_{2}}{2} \frac{\partial^{2} A}{\partial t^{2}}+\mathrm{i} \gamma|A|^{2} A+(G-\Gamma) A \\
& +\frac{G}{\Omega_{\mathrm{G}}^{2}} \frac{\partial^{2} A}{\partial t^{2}}+F_{\mathrm{SA}}\left(|A|^{2}\right) A,
\end{aligned}
$$

where $\beta_{2}$ and $\gamma$ are the dispersion and nonlinearity coefficients of the optical fiber averaged along the laser cavity, $\Omega_{\mathrm{G}}[\mathrm{THz}]$ is the spectral filtration parameter (the width of a parabolic spectral filter or the amplification band), and $G-\Gamma\left[\mathrm{dB} \mathrm{m}^{-1}\right]$ is the difference between the gain and losses in the cavity. We consider two important cases: (I) the constant amplification $G=G_{0}=$ const and (II) saturable amplification $G=$ $G(z)=G_{0} /\left(1+E(z) / E_{\text {sat G }}\right)$. In laser applications, the function $F_{\mathrm{SA}}\left(|A|^{2}\right)$ describes the distributed action of saturable absorber (5) in the effective simplified form. We list several of the most frequently used approximations.

(a) $F_{\mathrm{SA}}\left(|A|^{2}\right)=\delta|A|^{2}(\delta>0)\left[\mathrm{W}^{-1} \mathrm{~m}^{-1}\right]$ is the self-amplitude modulation coefficient describing the influence of a saturable absorber on a signal in the first nonlinear order. This equation is obtained from (8) by ignoring the third-order dispersion and the description of absorption saturation by the cubic term.

(b) $F_{\mathrm{SA}}\left(|A|^{2}\right)=\delta|A|^{2}\left(1-\zeta|A|^{2}\right)$. This approximation gives a generalized Ginzburg-Landau equation with the fifth-order nonlinear term (additionally to the third-order term) describing the self-amplitude modulation saturation. We consider it in detail in Section 3.3.

(c) $F_{\mathrm{SA}}\left(|A|^{2}\right)-\Gamma=-\Gamma_{0} /\left(1+|A|^{2} / P_{\text {sat }}\right)$ (where $P_{\text {sat }}$ is the saturation power in the saturable absorber). This represents the influence of the saturable absorber in the general form that follows from (5). In this case, the expansion of the term with saturable losses in a Taylor series gives rise to the thirdand fifth-order terms, as in cases (a) and (b).

We consider the most typical case of saturable amplification (II) and general expression (c) for a saturable absorber. Then the change in the signal energy is described by the equation

$$
\begin{aligned}
\frac{\mathrm{d} E}{\mathrm{~d} z} & =\frac{2 G_{0} E}{1+E / E_{\mathrm{sat}}}-2 \Gamma_{0} \int_{0}^{T_{\mathrm{R}}} \frac{|A|^{2} \mathrm{~d} t}{1+|A|^{2} / P_{\text {sat }}} \\
& -\frac{2 G}{\Omega_{\mathrm{G}}^{2}} \int_{0}^{T_{\mathrm{R}}}\left|A_{t}\right|^{2} \mathrm{~d} t .
\end{aligned}
$$

Depending on the compensation mechanism of the energy increase in Eqn (24), different classes of solutions of the generalized Ginzburg-Landau equation exist. The energy increase described by the first term in the right-hand side of (25) can be compensated by (a) linear decay (without absorption saturation, $\left.|A|^{2} / P_{\text {sat }} \ll 1\right)$; (b) losses related to the spectral filtration of the signal; (c) saturable losses depending on the field $|A|^{2}$; and a combination of these basic options.

We consider the limit with nonsaturable losses, $F_{\mathrm{SA}}\left(|A|^{2}\right)=0$, and the absence of the spectral filtration of amplification, $1 / \Omega_{\mathrm{G}}^{2}=0$. The evolution of the signal energy can then be found from the equation

$$
\frac{\partial E}{\partial z}=\frac{2 G_{0} E}{1+E / E_{\text {sat G }}}-2 \Gamma_{0} E
$$

The signal energy in the asymptotic limit is

$$
E_{\text {asym }}=E_{\text {sat G }}\left(\frac{G_{0}-\Gamma_{0}}{\Gamma_{0}}\right) \text {. }
$$

An analytic solution of Eqn (24) in the anomalous dispersion region has the form

$$
A(z, t)=\sqrt{\frac{\gamma E_{\text {asym }}^{2}}{-\beta_{2}}} \frac{1}{\cosh \left(T / T_{0}\right)} \exp \left(-\frac{\mathrm{i} \beta_{2} z}{2 T_{0}^{2}}\right),
$$

where $T_{0}=-\beta_{2} /\left(\gamma E_{\text {asym }}\right)$.

We now consider generalized Haus-Ginzburg-Landau equation (24) with $F_{\mathrm{SA}}\left(|A|^{2}\right)=\delta|A|^{2}(\delta>0)$ and spectral filtration. Then Eqn (24) has a solution in the form of a chirped dissipative soliton (with the time-dependent phase)

$$
A(z, t)=\sqrt{P} \operatorname{sech}^{1+\mathrm{i} C}\left(\frac{t}{\tau_{p}}\right) \exp (\mathrm{i} \phi z),
$$

where the parameters $P, \tau_{p}, C$, and $\phi$ are the respective signal peak power, duration, chirp, and phase. Substituting solution (29) in (24), we obtain the system of nonlinear algebraic equations

$$
\begin{aligned}
& \phi \tau_{0}^{2} \Omega_{\mathrm{G}}^{2}-\left[D\left(1-C^{2}\right)+2 C\right] \frac{\tau_{0}^{2}}{\tau_{p}^{2}}=0, \\
& D\left(2-C^{2}\right)+3 \bar{\gamma} \frac{\tau_{p}}{\tau_{0}}-3 C=0, \\
& \left(\frac{G_{0}}{1+E / E_{\mathrm{satG}}}-\Gamma\right) \frac{\Omega_{\mathrm{G}}^{2}}{G} \tau_{0}^{2}+\left[2 D C+\left(1-C^{2}\right)\right] \frac{\tau_{0}^{2}}{\tau_{p}^{2}}=0, \\
& \left(2-C^{2}\right)+3 D C-3 \bar{\delta} \frac{\tau_{p}}{\tau_{0}}=0 .
\end{aligned}
$$

Here, $\quad D=\beta_{2} \Omega_{\mathrm{G}}^{2} /(2 G), \quad \bar{\gamma}=\gamma \Omega_{\mathrm{G}}^{2} E \tau_{0} /(6 G), \quad$ and $\quad \bar{\delta}=$ $\delta \Omega_{\mathrm{G}}^{2} E \tau_{0} /(6 G)$ are dimensionless parameters, $\tau_{0}$ is the characteristic pulse duration, and $E=2 P \tau_{p}$ is the characteristic pulse energy. 

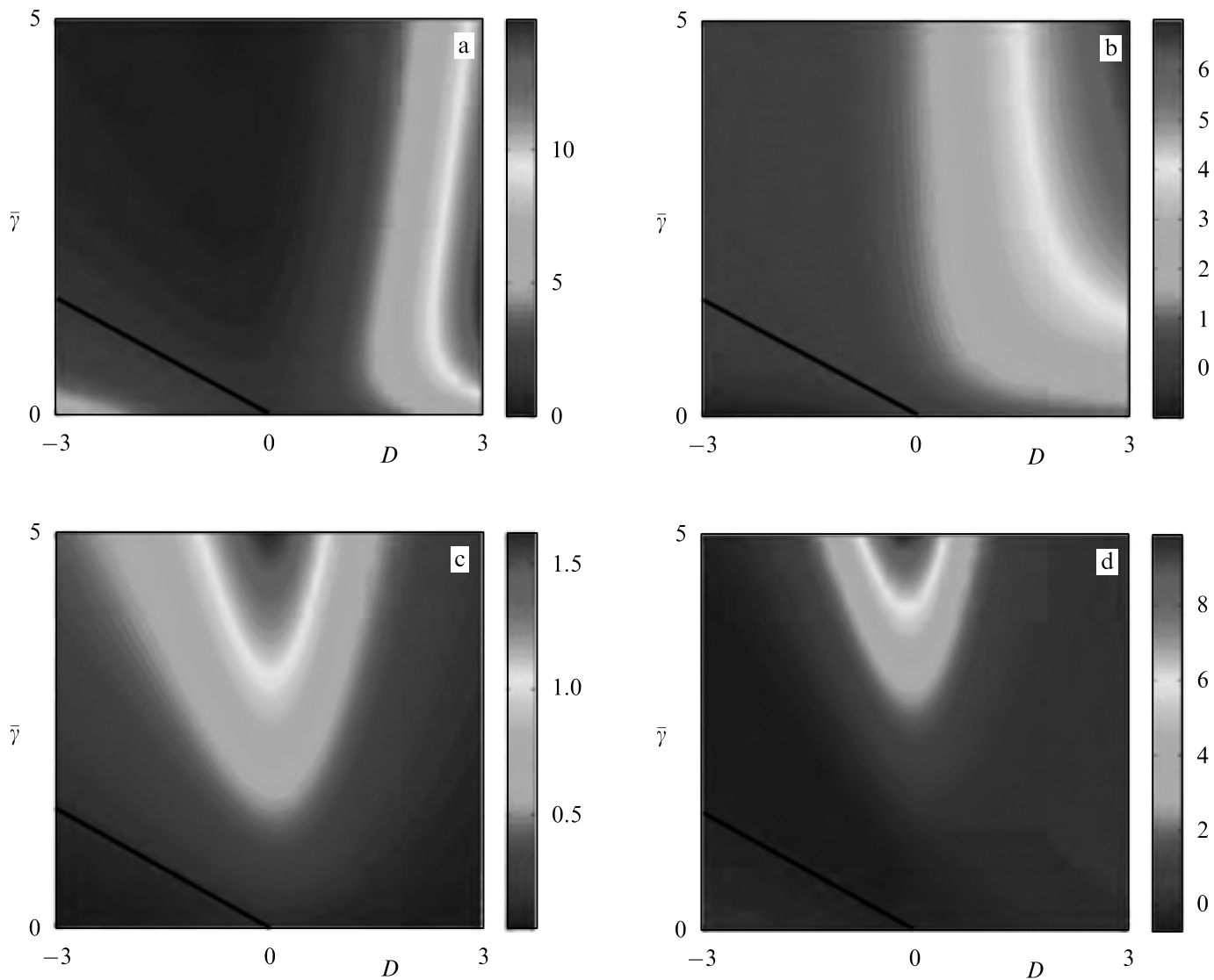

Figure 2. (Color online.) Solution of system of equations (30): (a) the duration $\tau_{p} / \tau_{0}$; (b) the chirp parameter $C$; (c) the spectral width $B$, (d) the gain parameter $(G-\Gamma) \Omega_{\mathrm{G}}^{2} \tau_{0}^{2}$ for the fixed parameter $\bar{\delta}=0.5$.

Figures 2 and 3 show the main characteristics of analytic solution (29), with exists in a broad range of dimensionless physical parameters (the figures are presented only in the limit $\left.E / E_{\text {sat G }} \ll 1\left(G=G_{0}\right)\right)$ [65]. The width of the signal spectrum is related to the signal duration as $B=$ $\tau_{0} \operatorname{arccosh}(\cosh (\pi C)+2) /\left(\pi^{2} \tau_{p}\right)$ [66-68]. In Fig. 2, the dispersion and Kerr nonlinearity coefficients $\beta_{2}$ and $\gamma$ change, whereas the signal energy $E$, the filter bandwidth $\Omega_{\mathrm{G}}$, and the self-amplitude modulation coefficient $\delta$ remain constant. If the nonlinearity parameter is nonzero, $\bar{\gamma} \neq 0$, a solution with zero chirp exists in the anomalous dispersion region on the straight line $D \bar{\delta}+\bar{\gamma}=0$ (black line). As a rule, pulsed solutions of Eqn (24) with the smallest duration and chirp exist in the anomalous dispersion region. Such solutions are similar to fundamental solitons, because they exist due to a balance between dispersion and nonlinearity, whereas the role of dissipative terms is secondary. However, unlike the nonlinear Schrödinger equation, the Ginzburg-Landau equation also has soliton solutions in the zero- and positivedispersion regions $(D \geqslant 0)$. Compared to fundamental solitons, pulsed solutions in the normal dispersion region have a longer duration and stronger chirp. The spectral width $B$ of the signal has a maximum in the vicinity of zero dispersion for $D>0$. The signal spectrum width increases as the nonlinearity parameter $\bar{\gamma}$ increases. The value of the gain $G-\Gamma$ determines whether the background noise in a laser increases or decreases. Figure $2 \mathrm{~d}$ shows that amplification is bounded in the anomalous dispersion region, whereas in the vicinity of zero dispersion $(D=0)$ for large nonlinearity parameters, the amplification begins to rapidly increase.
The influence of the amplification filtration on the form of the solution of Eqn (24) is illustrated in Fig. 3, ehich shows the main characteristic of the pulsed solution at the zero dispersion point $(D=0)$ for the fixed parameter $\Omega_{\mathrm{G}} \tau_{0}$ and energy $E$. The values of $\gamma \Omega_{\mathrm{G}}$ and $\delta \Omega_{\mathrm{G}}$ change. Rectangular regions I and II are the regions with strong and weak amplification filtering. If filtration is strong (the narrow gain bandwidth), the pulse duration and chirp parameter increase with increasing $\bar{\delta}$. In the case of weak filtration, the pulses become short and weakly chirped. In the entire region of dimensionless parameters, the signal spectral width increases with increasing the nonlinearity parameter $\bar{\gamma}$, while the gain decreases with increasing the self-amplitude modulation coefficient $\bar{\delta}$. Figure 4 presents the characteristic temporal and spectral profiles of solution (29) in the cases of normal and anomalous dispersion. The spectra of strongly chirped pulses in the normal dispersion region have a characteristic rectangular shape with sharp edges. Such solutions were first obtained experimentally in solid-state fiber lasers with normal mean dispersion [69].

In addition to the question of the existence of soliton solutions like (29), an important problem is the analysis of their stability. Figure $2 \mathrm{~d}$ shows the values of the gain parameter, which should be negative for a pulse to preserve its stability with respect to the increasing spontaneous noise in the cavity. Conditions providing the negative sign of the gain parameter were found in [42]. But the analysis performed in that paper does not allow completely characterizing the pulse stability in the cavity. Using the perturbation theory for solitons, the authors showed that solution (29) of Eqn (24) is 

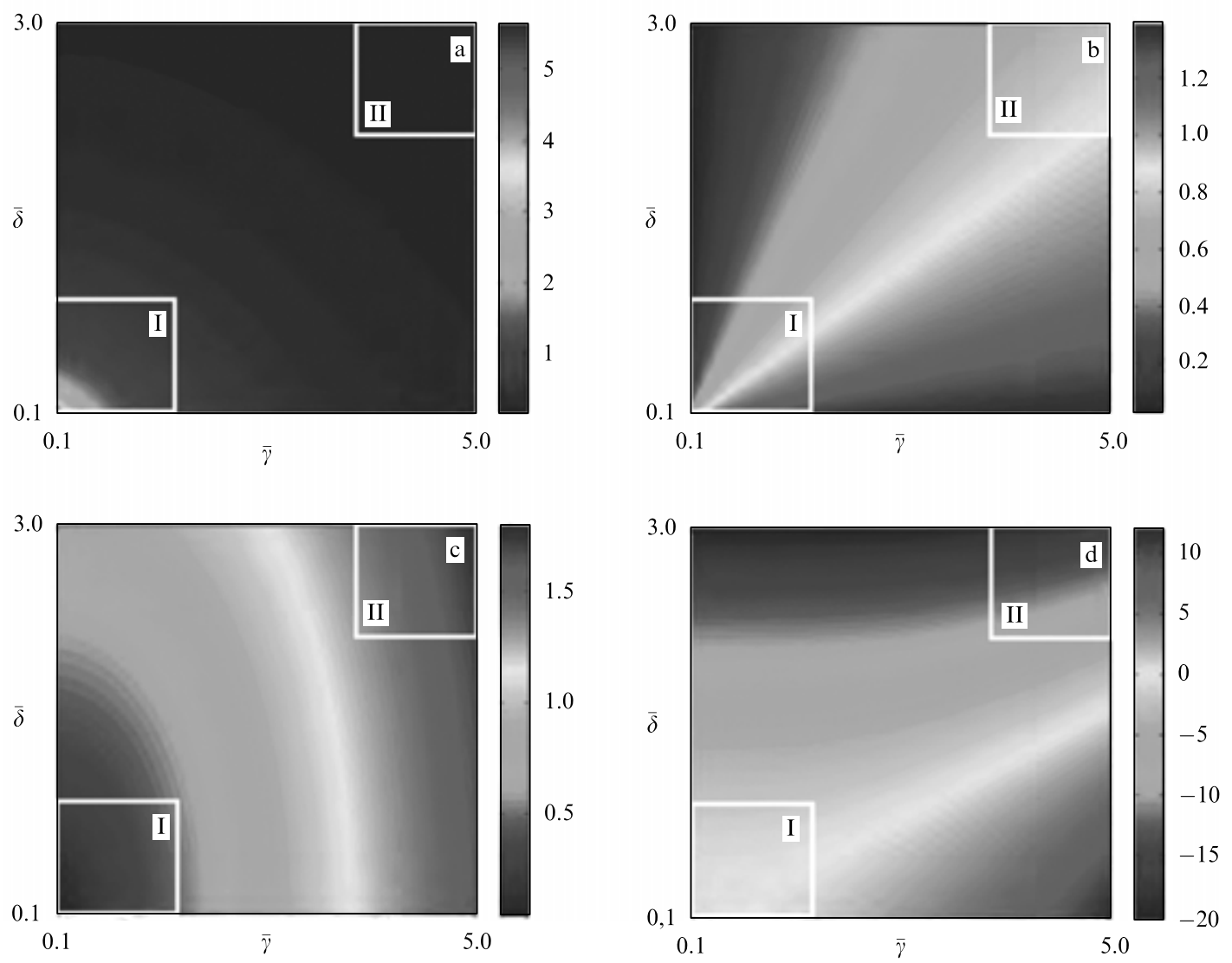

Figure 3. (Color online.) Solution of system of equations (30): (a) the duration $\tau_{p} / \tau_{0}$; (b) the chirp parameter $C$; (c) the spectral width $B$, (d) the gain parameter $(G-\Gamma) \Omega_{\mathrm{G}}^{2} \tau_{0}^{2}$ for the zero dispersion $D=0$.

unstable in the anomalous dispersion region $\left(\beta_{2}<0\right)$ if $G-\Gamma>0$ [42]. However, they neglected the amplification filtration and self-amplitude modulation. To analyze the stability more thoroughly, it is necessary to consider the saturable amplification (also see Eqn (18)):

$$
G=G(z)=\frac{G_{0}}{1+E(z) / E_{\mathrm{sat} \mathrm{G}}} .
$$

In denving the model of saturable amplification in Eqn (31), it was assumed that the response of the medium is slow compared to the round-trip time $T_{\mathrm{R}}$ of a pulse in the cavity. The time response of the medium is typically about a microsecond, whereas $T_{\mathrm{R}}$ is of the order of a nanosecond in many optical systems, including fiber laser cavities. The model with saturable amplification stabilizing the signal energy and restricting its growth allows finding stable solutions of Eqn (24) in the normal $\left(\beta_{2}>0\right)$ and anomalous $\left(\beta_{2}<0\right)$ dispersion regions [70].

Ginzburg-Landau equations (24) with the cubic approximation of the saturable absorber $\left(F_{\mathrm{SA}}\left(|A|^{2}\right)=\delta|A|^{2}\right.$ describe the main characteristics of soliton solutions like (29); however, the region of existence of stable solutions found from this equations is bounded. Broader classes of stable soliton solutions appear when the fifth-order term describing the saturation of the self-amplitude modulation is taken into account. The equation obtained in this way is called the generalized Ginzburg-Landau equation. We note that chirped soliton pulses like (29) are no longer exact solutions of the new equation. Nevertheless, the main dependences shown in Figs 2 and 3 remain valid for stable solutions of the
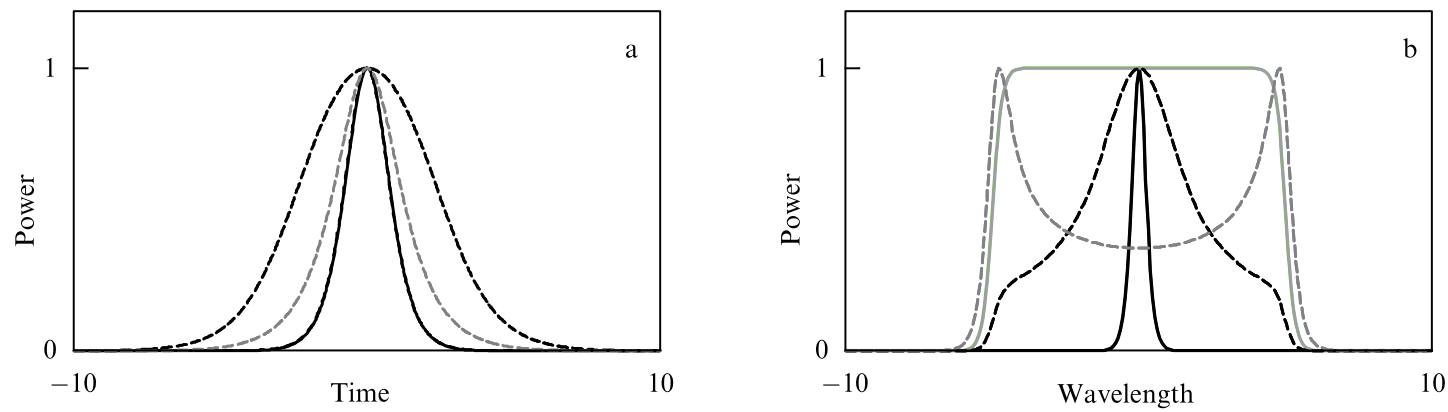

Figure 4. (a) Temporal and (b) spectral profiles of the solution in the form of a hyperbolic secant for $P=1, \tau_{p}=1, C=0$ (solid black line), and $C=5$ (solid grey line). Dashed curves show strongly chirped solutions of the generalized Ginzburg-Landau equation. 
generalized Ginzburg-Landau equation. For example, pulses in the anomalous dispersion region have a small chirp and are short compared to long strongly chirped pulses in the normal dispersion region. To date, numerous papers devoted to the search for and study of the behavior of solutions of the generalized Ginzburg-Landau equation have been published. The subjects of the studies are, for example, stationary solutions, pulsed solitons, and the interaction and destruction of solitons [9, 71-74].

Recent progress in the study and development of fiber lasers and oscillators with completely normal dispersion resulted in the generation of strongly chirped dissipative solitons with high energies [75, 76]. Some properties of strongly chirped dissipative solitons cannot be described by the cubic Ginzburg-Landau equation. For example, the spectrum of such pulses can differ from the spectrum of solution (29). Examples of such spectra are presented in Fig. 4 (dashed curves). The spectra have a parabolic upex or a dip in the middle and sharp edges. The solution for the generalized Ginzburg-Landau equation with the fifth-order nonlinear term

$$
\begin{aligned}
A(z, t) & =\sqrt{\frac{P}{\cosh \left(t / \tau_{p}\right)+B}} \\
& \times \exp \left[-\mathrm{i} C\left(\ln \left(\cosh \frac{t}{\tau_{p}}+B\right)\right)+\mathrm{i} \phi z\right]
\end{aligned}
$$

reproduces the complex shape of the spectrum, depending on the value of the parameter $-1<B<\infty$ [77]. To obtain the solution in this form, it is necessary to make some assumptions about the parameters of the equation. However, this solution qualitatively describes different lasing regimes in fiber lasers with completely normal dispersion, including rectangular pulses, strongly chirped dissipative solitons, and multipulse lasing regimes. It is also used to describe the intracavity dynamics of the signal and the influence of spectral filtration on signal generation in fiber lasers [78]. Recently, this solution was used to generalize the so-called area theorem. Unlike the standard soliton area theorem, which states that the soliton energy is inversely proportional to its duration, the pulse energy in the generalized theory for a certain set of parameters linearly increases with the pulse duration [79].

The basic idea of another approach is the search for approximate solutions of the generalized Ginzburg-Landau equation qualitatively repeating the features of experimental pulses $[64,80-82]$. These solutions can be assigned to a certain set of parameters and generalized to the case where dissipative third- and fifth-order terms in the equation are replaced by a Lorentzian, as in Eqn (14) [83].

\subsection{Automodel solutions}

It was found in [84] that high-power parabolic optical pulses with a linear frequency chirp can stably propagate in a passive fiber with positive dispersion. This observation aroused interest in the study of the propagation of short pulses in fiber amplifiers with positive (normal) dispersion, which are described by Eqn (24) for $\beta_{2}>0, \Omega_{\mathrm{G}} \rightarrow \infty$, and $\delta=0$. We note that independently of the initial pulse shape, the pulse becomes parabolic during its propagation in the fiber and acquires a quadratic phase if $G-\Gamma>0$ [85]. Figure 5a shows the evolution of an initially Gaussian beam propagating in a fiber. The inset shows the pulse after propagation, which has a characteristic parabolic temporal profile and a linear chirp (the derivative of the phase). Indeed, irrespective of the initial signal shape, the chirped parabolic pulse is a global attractor for the signal in the asymptotic limit. We represent the automodel parabolic solution in the form

$$
A(z, t)=\sqrt{P_{p}(z)\left[1-\frac{t^{2}}{\tau_{p}^{2}(z)}\right]} \exp \left(\mathrm{i} C_{p}(z) t^{2}\right) .
$$

Substituting (32) in (24) (with $\Omega_{\mathrm{G}} \rightarrow \infty$ and $\delta=0$ ) and assuming that $1 / \tau_{p}^{2} \ll C_{p}^{2}$ (the so-called semiclassical approximation), we obtain in the asymptotic limit

$$
\begin{aligned}
& \frac{\mathrm{d} P_{p}}{\mathrm{~d} z}=2 \beta_{2} P_{p} C_{p}+2(G-\Gamma) P_{p}, \\
& \frac{\mathrm{d} \tau_{p}}{\mathrm{~d} z}=-2 \beta_{2} \tau_{p} C_{p}, \\
& \frac{\mathrm{d} C_{p}}{\mathrm{~d} z}=2 \beta_{2} C_{p}^{2}-\gamma \frac{P_{p}}{\tau_{p}^{2}} .
\end{aligned}
$$

Equation (33) has the analytic solutions

$$
\begin{aligned}
P_{p}(z) & =A_{0}^{2} \exp \frac{4(G-\Gamma) z}{3}, \\
\tau_{p}(z) & =\frac{3 \sqrt{\gamma \beta_{2} / 2}}{G-\Gamma} A_{0} \exp \frac{2(G-\Gamma) z}{3}, \\
C_{p}(z) & =-\frac{G-\Gamma}{3 \beta_{2}}
\end{aligned}
$$
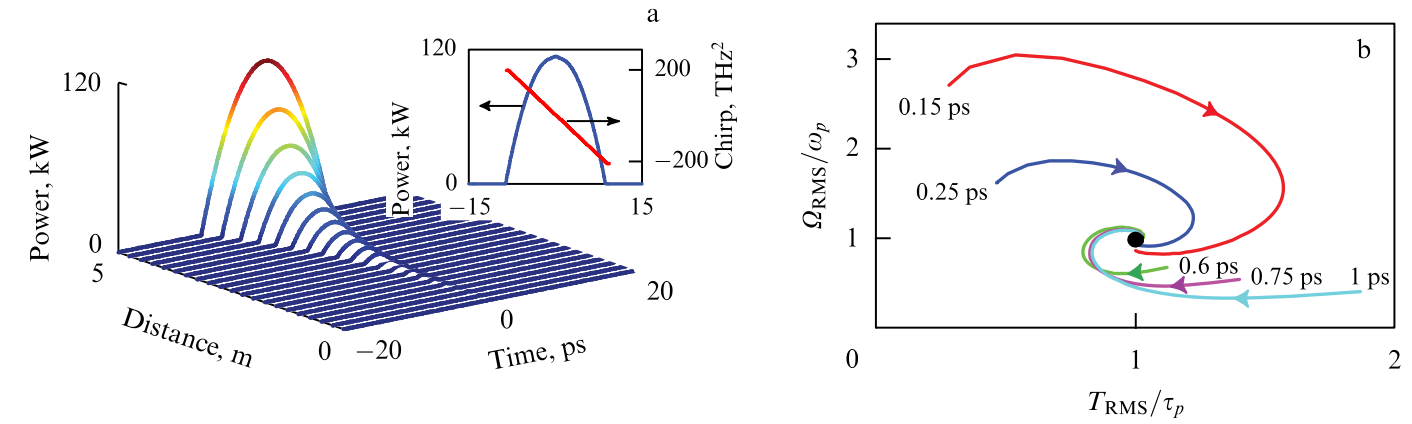

Figure 5. (Color online.) (a) Solution of Eqn (24) with a Gaussian pulse as the initial signal. The initial pulse energy is $E=100$ pJ, the pulse duration is $T_{\mathrm{FWHM}}=500 \mathrm{fs}$, the fiber length is $L=5 \mathrm{~m}, \beta_{2}=0.025 \mathrm{ps}^{2} \mathrm{~m}^{-1}, \gamma=0.0051 \mathrm{~W}^{-1} \mathrm{~m}^{-1}, G=0.95 \mathrm{~m}^{-1}, \Gamma=\delta=0, \Omega \rightarrow \infty$. The inset shows the power and chirp of a pulse propagating in the fiber. (b) Phase portraits of solutions corresponding to the initial pulse duration varying from $150 \mathrm{fs}$ to 1 ps. 
where $A_{0}=\left[2(G-\Gamma) E_{\text {in }} / \sqrt{\gamma \beta_{2} / 2}\right]^{1 / 3} / 2$ and $E_{\text {in }}$ is the signal energy at the fiber input. The spectral width of the signal can be found from Eqn (34) and written in the form $\omega_{p}=$ $\sqrt{2 \gamma /\left(5 \beta_{2}\right)} A_{0} \exp [2(G-\Gamma) z / 3]$. If the gain is positive $(G-\Gamma>0)$, the signal peak power, duration, and spectral width increase exponentially and depend only on the amplifier parameters and the initial energy. The signal chirp during pulse propagation in the fiber becomes constant, being proportional to the gain and inversely proportional to the group velocity dispersion coefficient. The pulse characteristics asymptotically approach solution (34) independently of its initial shape. Figure $5 \mathrm{~b}$ shows the phase plane determined by the ratio of the signal RMS duration and spectral width to the respective asymptotic values $\tau_{p}$ and $\omega_{p}$. The results are obtained by solving Eqn (24) with the Gaussian pulse $A(0, t)=\left\{E_{\text {in }} /\left[\sqrt{\pi} \tau_{p}(0)\right]\right\}^{1 / 2} \exp \left[-t^{2} /\left(2 \tau_{p}^{2}(0)\right)\right]\left(E_{\text {in }}=100 \mathrm{pJ}\right)$ as the initial field. The pulse duration (FWHM) was changed from $150 \mathrm{fs}$ to $1 \mathrm{ps}$; the amplifier length was $5 \mathrm{~m}$. Although the evolution of pulses with different initial durations proceeds along different trajectories, all the pulses tend to the asymptotic solution in the form of a similariton (a point with coordinates $(1,1)$ in the phase plane). Because the above solutions, called similaritons, are global attractors for the system, they can find applications in various optical systems.

\subsection{Other analytic solutions}

We now consider practically important solutions (PodivilovKalashnikov solitons [64]) of the complex generalized Ginzburg-Landau equation (cubic-quintic GinzburgLandau equation, CQGLE) for an envelope that differs from the usual Haus-Ginzburg-Landau equation (24) by a higher-order nonlinear term:

$$
\begin{aligned}
\frac{\partial A}{\partial z} & =\frac{\mathrm{i} \beta_{2}}{2} \frac{\partial^{2} A}{\partial t^{2}}-\mathrm{i} \gamma|A|^{2} A+(G-\Gamma) A \\
& +\frac{G}{\Omega_{\mathrm{G}}^{2}} \frac{\partial^{2} A}{\partial t^{2}}+\delta|A|^{2}\left(1-\zeta|A|^{2}\right) A .
\end{aligned}
$$

For convenience, we set $\sigma=\Gamma-G$ and $\eta=G / \Omega_{\mathrm{G}}^{2}$.

For strongly chirped dissipative solitons, the first two terms describing the dispersion $\left(\beta_{2}\right)$ and self-phase modulation (SPM) $(\gamma)$ are much greater than the remaining four terms describing the difference between losses and amplification $(\sigma)$, the spectral filtration $(\eta)$, the self-amplitude modulation $\delta$, and the saturation of self-amplitude modulation $(\zeta)$. All the parameters are positive for the chosen notation.

The pulse shape $A(t)$ averaged along the cavity can be found by solving Eqn (35) under the stationarity condition $\partial A / \partial z=0$. An exact analytic solution of the stationary CQGLE for the normal dispersion was first found in [86] in the case of weakly chirped solitons. An analysis of the stability and determination of the existence region for this solution were presented in $[71,72,87]$. To characterize the pulse chirp (phase modulation), the exact solution [86] can be written in the form $A=a(t)^{1+\mathrm{i} f}$ [72], where $f$ is the dimensionless chirp parameter. The strong-chirp limit $f \rightarrow \infty$ is achieved when the spectral filtration in the system is much smaller than the total dispersion $\left(\eta \ll \beta_{2}\right)$ and the selfamplitude modulation is much smaller than the self-phase modulation $(\delta \ll \gamma)$. In this limit, the chirp parameter is expressed in terms of coefficients describing the cavity parameters: $f \simeq 3 /\left(\eta / \beta_{2}+\delta / \gamma\right) \gg 1$ (see, e.g., [72]). In this case, the parameter $f$ is equal to the half-width $\Delta$ of the spectrum times the pulse FWHM $\tau_{p}\left(f \simeq \Delta \tau_{p}\right)$. The exact solution [86] for a large chirp becomes singular and has no physical. Because of this, the universal approach to the analysis of strongly chirped dissipative solitons based on the stationary phase method was presented in [64].

According to the approach in [64], the peak pulse power $P_{m}$ can take two values:

$$
P_{m}^{ \pm}=\frac{3}{8 \zeta}\left(2-\frac{\eta \beta_{2}}{\delta \gamma} \pm \sqrt{\left(2-\frac{\eta \beta_{2}}{\delta \gamma}\right)^{2}-16 \frac{\sigma \zeta}{\delta}}\right) .
$$

The expression describing the pulse shape can be written implicitly [64] for both positive $\left(P_{m}=P_{m}^{+}\right)$and negative $\left(P_{m}=P_{m}^{-}\right)$branches:

$$
\operatorname{arctanh}\left(\frac{\Omega(t)}{\Delta}\right)+\frac{1}{R} \arctan \left(\frac{\Omega(t)}{R \Delta}\right)=\frac{t}{\tau_{p}} .
$$

Here, $\Delta=\sqrt{\left(\gamma / \beta_{2}\right) P_{m}}$ is the half-width of the spectrum of a strongly chirped dissipative soliton, $\tau_{p}=$ $3 \gamma^{2} /\left(\beta_{2} \zeta \delta \Delta^{3}\left(1+R^{2}\right)\right)$ is the pulse duration, $\Omega(t)$ is the instant frequency, and

$$
R=\sqrt{\frac{1+\eta \gamma / \beta_{2} \delta}{\zeta P_{m}}-\frac{5}{3}} .
$$

is the only parameter determining the pulse shape. The spectrum is described by

$$
I(\omega) \simeq \frac{6 \pi \gamma}{\zeta \delta} \frac{H\left(\Delta^{2}-\omega^{2}\right)}{\omega^{2}+R^{2} \Delta^{2}},
$$

where $H(x)$ is the Heaviside function. We can see that the shape of the spectrum depends only on the parameter $R$.

A remarkable feature of this solution is its scalability. The pulse shape $P(t) / P^{ \pm}=B\left(t / \tau_{p}\right)$ and the shape of its spectrum $I(\omega) / I(0)=C(\omega / \Delta)$ depend only on the parameter $R$. If all six parameters of the initial equation are changed such that the value of $R$ is preserved, the pulse shape and the shape of its spectrum do not change. The pulse shape is preserved, while its amplitude, duration, chirp, and spectral width are scaled.

In the limit case $\zeta P_{m} \ll 1$, the solution $P(t)$ tends to $\operatorname{sech}^{-2}\left(t / \tau_{p}\right)$. Obviously, in this case the self-amplitude modulation (cubic term) is small. We note that in the opposite limit $R \rightarrow 0$, the pulse shape approaches a rectangular one. The chirp parameter for $R \rightarrow \infty$ can be written in the form

$$
f=\Delta \tau_{p}=3\left(\frac{\delta}{\gamma}+\frac{\eta}{\beta_{2}}-\frac{2}{3} \zeta P_{m} \frac{\delta}{\gamma}\right)^{-1},
$$

which is close to the exact solution [72]. We note that the expression for the chirp parameter in the opposite limit can be considerably different.

Because the pulse power can take only positive real values, it follows from (36) that solutions are absent in the region $\eta \gamma / \beta_{2} \delta<2-4 \sqrt{\sigma \zeta / \delta}$. The domain of definition of the analytic solution is shown in Fig. 6 in the plane $\left(\eta \gamma / \beta_{2} \delta, \sigma \zeta / \delta\right)$. The region where solutions are absent is denoted by I. Another condition follows from restrictions on the pulse spectrum: $R^{2}>0$. For the positive branch $\left(P_{m}=P_{m}^{+}\right)$, solutions exist in region IV, while for the negative branch $\left(P_{m}=P_{m}^{-}\right)$, they are in regions III and IV. In region II, both solutions are absent because the condition $R^{2}<0$ is satisfied there. In this case, the negative branch is unstable in the entire region of existence (regions III and IV), 

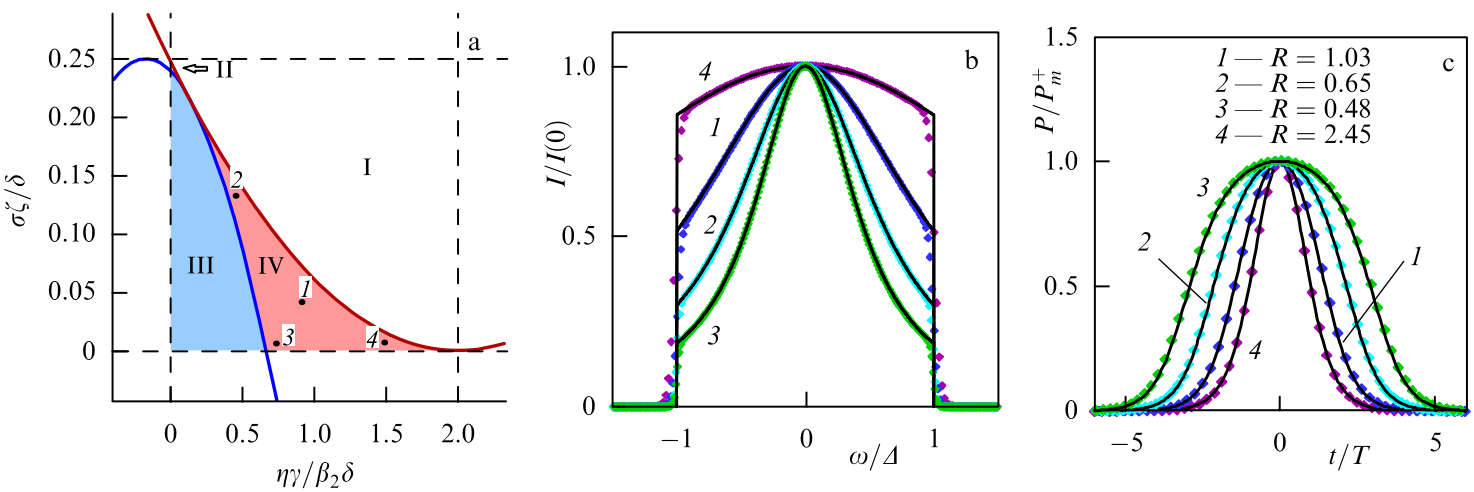

Figure 6. (Color online.) (a) Region of the solution existence in the $\eta \gamma / \beta_{2} \delta, \sigma \zeta / \delta$ plane; the positive branch $\left(P_{m}=P_{m}^{+}\right)$is defined in region IV, the negative branch $\left(P_{m}=P_{m}^{-}\right)$is defined in regions III and IV. Points at which numerical calculations were performed are indicated by the numbers $1-4$. (b) Comparison of numerical calculations (symbols) with the positive branch of the analytic solution (solid dark line) for pulse spectra. (c) The pulse shape in the temporal space for the positive branch of the analytic solution (solid lines)

while the positive branch is stable in its own region of existence (region IV). In region I, stable solutions exist for a weak chirp $f \leqslant 1$, whereas strongly chirped solutions are absent in this region. In region III, stable solutions have not been obtained for either a strong or a weak chirp. Thus, region IV is the only region of existence and stability for strongly chirped solutions of Eqn (35).

We now compare numerical and analytic solutions for different points in region IV in Fig. 6a. Numerical simulation was performed for the following points: point $1(\sigma=0.04$, $\eta=1, \gamma=9)$, point $2(\sigma=0.13, \eta=0.5, \gamma=10)$, point 3 $(\sigma=0.006, \eta=0.75, \gamma=10)$, and point $4(\sigma=0.007$, $\eta=1.25, \gamma=12)$. Parameters $\beta_{2}=10, \delta=1$, and $\zeta=1$ were not changed.

Calculations showed that the results of numerical simulation for selected points coincide with the analytic solution for the positive branch in the entire domain of definition (Fig. 6b). This means that the spectral shape of the strongly chirped dissipative soliton solution in Eqn (35) generally depends only on the parameter $R$ and is independent of other parameters of the equation.

Figure $6 \mathrm{c}$ presents pulse shapes in the temporal region at different points in the domain of definition. The solution shape is shown for different values of $R$. For example, for $R=2.45$, the pulse shape is well approximated by the function $\mathrm{sech}^{-2}$, which is close to the standard soliton solution of the Ginzburg-Landau equation. As $R$ decreases, the pulse shape changes and approaches a parabolic one at point 3 (near the left boundary of region IV in Fig. 6a). For $R \rightarrow 0$ (exactly on the left boundary of region IV), the pulse shape is close to rectangular: $P=P_{m}^{+}\left(1-R^{2} \tan ^{2}\left(R t / \tau_{p}\right)\right)$ for $t<\left(\tau_{p} / R\right) \pi / 2$.

\section{Poincaré map in describing laser systems}

The averaged models considered above are invalid when changes in the optical pulse amplitude and (or) phase after the round trip of light in the cavity become considerable. Modern mode-locked fiber lasers generating ultrashort highenergy pulses usually exhibit strongly nonlinear dynamics during a round trip, corresponding to considerable changes in the pulse shape. For example, during the propagation of a high-energy pulse in an optical fiber, the pulse spectrum can be considerably broadened after a round trip in the cavity, violating the condition on which the averaged models of mode-locked fiber lasers are based. In this case, the modified Haus-Ginzburg-Landau equation can be used only to simulate the propagation of the optical field through active and passive fibers. Other discrete elements, such as a saturable absorber, a coupler, and filters, should be considered separately from the pulse propagation in the fiber and should not be included in the distributed model. In the case of stable lasing, the generated pulse should be reproduced after each round trip in the cavity. Mathematically, this corresponds to the so-called Poincaré map, which is more convenient for describing the periodic laser dynamics than the averaged model is. The total change $\widehat{T}_{\mathrm{RT}}$ in the optical field for a round trip in the laser cavity is the result of successive transformations of the field in each element of the laser system: $\widehat{T}_{\mathrm{RT}}=\widehat{T_{1}} \widehat{T_{2}} \widehat{T_{3}} \widehat{T_{4}} \ldots$. Here, $\widehat{T_{k}}$ is the complex transformation operator acting on the optical field in different intracavity elements, such as passive and active fibers, an outcoupler, a saturable absorber, and filters. The total field transformation $A(t)$ after a round trip in the cavity is given by the operator $\widehat{T}_{\mathrm{RT}}: A_{n+1}=\widehat{T}_{\mathrm{RT}} A_{n}$. In the simplest case, the asymptotic state corresponds to a fixed point of this Poincare map: $A_{n+1}=\widehat{T}_{\mathrm{RT}} A_{n}=\exp (\mathrm{i} \Lambda) A_{n}$. The inclusion of discrete elements in the laser simulation directly rather than in the form averaged over many periods emphasizes the periodic nature of the radiation dynamics in the cavity. The intracavity dynamics can be optimized by changing the system parameters or the laser configuration itself. Mathematically, the nonlinear dynamics of light in the laser cavity makes the operators $\widehat{T_{k}}$, transforming the field in isolated point-like elements, noncommuting. A significant practical consequence of this mathematical fact is the importance (for the properties of generated pulses) of the order in which pointlike elements are arranged in the laser cavity.

For example, we consider the typical setup of a dissipative soliton fiber laser with normal dispersion everywhere and a ring cavity consisting of an active fiber (AF), a passive fiber (PA), a saturable absorber (SA), and an outcoupler (OC). We compare the pulse energy at the system output (behind the coupler) for different variants of the arrangement of elements in a fiber laser of this type. We let each configuration be denoted by a sequence of components according to their arrangement in the cavity. For example, the configuration $\mathrm{AF}-\mathrm{PF}-\mathrm{SA}-\mathrm{OC}$ means that the active fiber is followed by the passive fiber, then by the saturable absorber, and the output coupler. For convenience, each configuration ends with the 
outcoupler. Hence, for the set of elements listed above, six different configurations are possible. In the order of decreasing the output energy, they are (1) PA-AF-SA-OC; (2) AFSA-PF-OC; (3) AF-PF-SA-OC; (4) SA-PF-AF-OC; (5) PF-SA-AF-OC; (6) SA-AF-PF-OC. Thus, to achieve the maximum output energy from the cavity, it is necessary to place the saturable absorber behind the amplifying medium and the outcoupler behind the saturable absorber [88].

The advantage of the configuration providing the maximum output energy becomes more substantial with increasing the cavity length. For short cavities, the relation

$$
E_{\text {out }}^{(1)} \approx E_{\text {out }}^{(2)} \approx E_{\text {out }}^{(3)}>E_{\text {out }}^{(4)} \approx E_{\text {out }}^{(5)} \approx E_{\text {out }}^{(6)}
$$

is valid, where $E_{\text {out }}^{(i)}$ is the output energy from the cavity with the $i$ th configuration of elements. Changes in the energy and other parameters of generated pulses upon changing the order of intracavity elements most clearly demonstrated the nonlinear character of the appearing structures.

There are two types of scales in lasers: the 'fast' scale corresponding to the pulse dynamics at the cavity scale and the 'slow' scale corresponding to pulse changes for many round trips in the cavity. The slow evolution can be described using different mathematical approaches leading to HausGinzburg-Landau equations. Such slow dynamics can be visualized at a chosen point in the cavity via the 'stroboscopic' evolution or by considering the evolution in terms of the Poincaré map in periodic systems.

We consider several examples. Figure 7 a shows the typical phase portrait in the chirp-pulse-width plane for a SESAM (semiconductor saturable absorber mirror) passively modelocked fiber laser described in detail in Section 8.2. The plot was obtained by using the following parameters of the fiber laser: the gain $g_{0}=5.5 \mathrm{~dB}$, the second-order cumulative dispersion $\beta_{2}=-0.046 \mathrm{ps}^{2}$, the third-order dispersion $\beta_{3}=$ $5 \times 10^{-4} \mathrm{ps}^{3}$, the modulation depth $q_{0}=0.3$, the saturation energy $E_{\mathrm{sat}}=0.5 \mathrm{pJ}$, and the SESAM recovery time $\tau_{\text {sat }}=10$ ps. We can see that the initial noise perturbation evolves to the single-pulse regime with the following asymptotic parameters: the pulse duration $T_{\mathrm{FWHM}}=0.56 \mathrm{ps}$, the chirp parameter $C=-3.44 \mathrm{ps}^{-2}$, and the peak power $P_{0}=120 \mathrm{~W}$. The mathematical solution is an attractor, and any initial distribution tends to this stable asymptotic state.
Figure $7 \mathrm{~b}$ shows the dependence of attractors on the initial conditions for a dissipative soliton fiber laser with a cavitylength of $2002 \mathrm{~m}$, whoseschemeis presented in Section 8.1. The pulse parameters (its RMS width and power) are presented at the output of the laser cavity. One of the lines corresponds to the evolution of the initial Gaussian field distribution and the other to the evolution of white noise to the attractors indicated by circles.

Because the laser is a dissipative system, it can be seen that in both cases the evolution of the initial field distribution goes along a spiral to the attraction point - a stable pulse corresponding to a periodic solution.

\section{Simplified models describing the dynamics of laser pulses}

The pulse dynamics in a fiber can often be described by evolutionary equations for the pulse duration, peak power, energy, the chirp parameter, and the spectral width [41]. Under some assumptions, the particle-like properties of pulses allow obtaining a closed system of coupled ordinary differential equations (ODEs) well approximating the key features of the pulse dynamics. This important simplification is a direct consequence of the fact that the pulse is a wave packet that can be described well by a finite number of degrees of freedom. Instead of analyzing partial differential equation (24), the pulse dynamics can described by a finite system of ODEs. For the NSE, such ODEs are obtained by the variational method or the method of moments (ideologically close to the Galerkin method). The variational method [89] is based on the possibility of rewriting the NSE in terms of a variational problem in which the Lagrangian should be minimized for the envelope function. This was first used for the NSE by Anderson in 1983 [90] and was then extensively applied for simulating various NSE systems (see, e.g., [91-95]). The method of moments (or the root-mean-square (RMS) method of moments), first demonstrated in nonlinear optics in 1971 [96], allows calculating the pulse duration and peak power in terms of time-integrated quantities. The master partial differential equation can be transformed into an ODE system describing the dynamics of integrated characteristics over $z$ [97] (RMS). This method is used to describe the propagation of
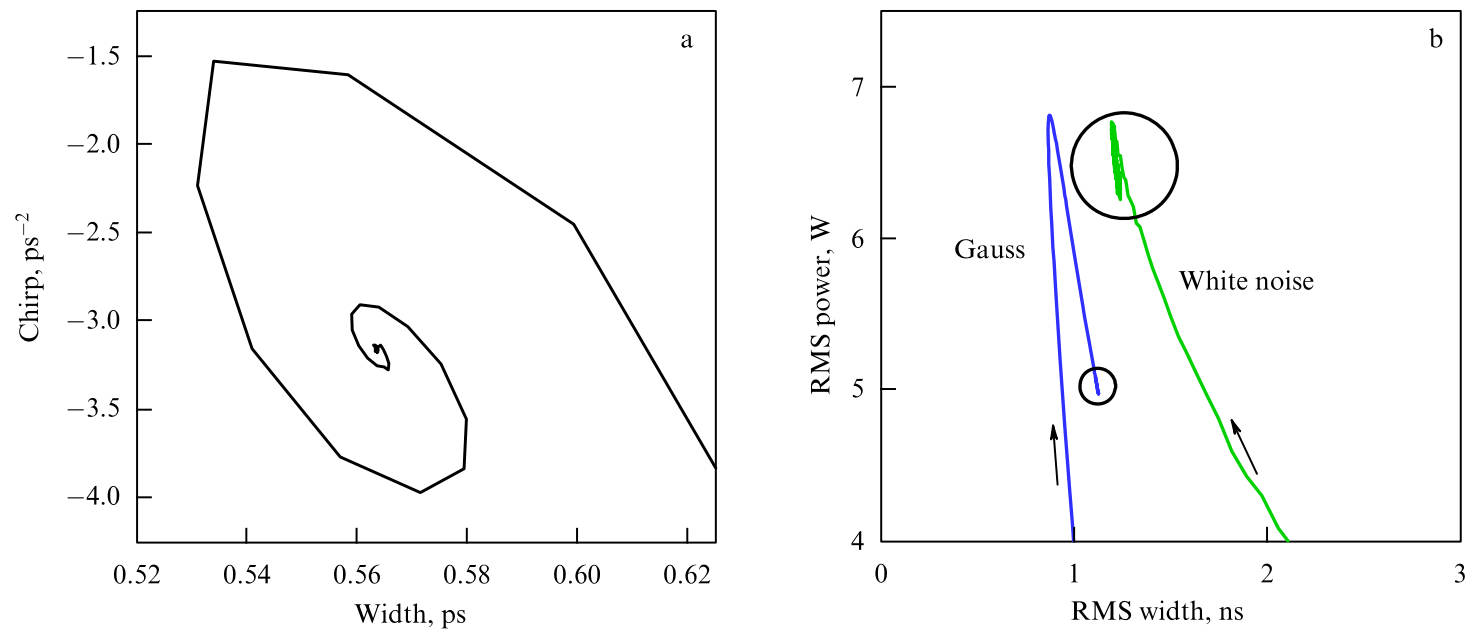

Figure 7. (a) Phase portrait of a pulse in the chirp-pulse-width plane. (b) Attractors for different initial field distributions: the evolution of the initial Gaussian distribution and white noise to the attractors indicated by circles. 
pulses in fibers [98, 99] and dispersion-controlled systems [94, 100-103].

The most popular approach to the mathematical simulation of the generation and propagation of optical pulses in modern fiber laser systems is the successive solution of equations corresponding to each element of the cavity (see, e.g., $[9,28,59,61,65,104-109])$. In general, it is necessary to perform separate calculations for each particular set of the system parameters and to monitor the pulse dynamics during many round trip in the cavity until pulse stabilization. This approach often provides a high calculation accuracy and good agreement with experimental data. However, any investigations involving multiparametric optimization of the fiber laser design are time consuming and require high-power computers. In the case of multiparametric optimization, the most efficient are methods assuming the lower accuracy due to the use of some analytic approximations and the distributed consideration of the action of optical devices in the laser, and also the use of relatively fast algorithms for numerical calculations. The shape of stable lasing regimes in fiber lasers (soliton regimes) with dispersion control are often characterized by the automodel central part and decaying tails $[65,94,100,101,103,110,111]$, the difference between the total pulse energy and the energy of its central part being insignificant. In this connection, of most interest is the study of the 'fast' dynamics (within one round trip in the cavity) of the central part of optical pulses. Such a division of the dissipative soliton shape into the automodel central part and decaying tails allows the use of simpler mathematical models based on the systems of ordinary nonlinear differential equations for studying the dynamics of optical pulses in laser cavities.

The propagation of optical pulses in an active optical fiber can be described by the generalized NSE $[9,28,59,61,65$, 104-109] [cf. Eqn (24)]:

$$
\frac{\partial A}{\partial z}=-\frac{\mathrm{i} \beta_{2}}{2} \frac{\partial^{2} A}{\partial t^{2}}+\mathrm{i} \gamma|A|^{2} A+(G-\Gamma) A+\frac{G}{\Omega_{\mathrm{G}}^{2}} \frac{\partial^{2} A}{\partial t^{2}},
$$

where, in the general case, $G=G(z)$ and obeys Eqn (31).

We consider stable single-pulse lasing regimes. With this condition satisfied, pulses have already produced the amplification saturation after previous round trips in the cavity and a balance is established between amplification and losses. In this case, a relation of unsaturated amplification to the pulse energy can be defined in terms of the parameters $\Gamma, P_{\text {sat G }}$, and $T_{\mathrm{R}}$ of a fiber laser.

We introduce the notation $\tilde{z}=z / L$, where $L$ is the laser cavity length. We then obtain the scaled equation

$$
A_{\tilde{z}}=\mathrm{i} d(\tilde{z}) A_{t t}+\mathrm{i} \varepsilon|A|^{2} A+\left(g-l_{0}\right) A+v g A_{t t},
$$

where $d(\tilde{z})=-\beta_{2} L / 2, \varepsilon=\gamma L, v=G / \Omega_{\mathrm{G}}^{2}, l_{0}=\Gamma L, g=G L$. After scaling, we have $d(\tilde{z}+1)=d(\tilde{z})$.

Equation (41) can be transformed into a system of equations for the RMS characteristics of the pulse [65, 108]. To describe the dynamics of the central part of a soliton with the help of key characteristics of the pulse, we consider the evolution of the integrated quantities $[41,52,99,112]$

$$
T_{\mathrm{RMS}}(\tilde{z})=\sqrt{\frac{\int_{0}^{T_{\mathrm{R}}} t^{2}|A(\tilde{z}, t)|^{2} \mathrm{~d} t}{\int_{0}^{T_{\mathrm{R}}}|A(\tilde{z}, t)|^{2} \mathrm{~d} t}},
$$

$$
\begin{aligned}
& C_{\mathrm{RMS}}(\tilde{z})=\frac{\mathrm{i}}{4} \frac{\int_{0}^{T_{\mathrm{R}}} t\left[A(\tilde{z}, t) A_{t}^{*}(\tilde{z}, t)-A^{*}(\tilde{z}, t) A_{t}(\tilde{z}, t)\right] \mathrm{d} t}{\int_{0}^{T_{\mathrm{R}}}|A(\tilde{z}, t)|^{2} \mathrm{~d} t}, \\
& P_{\mathrm{RMS}}(\tilde{z})=\frac{\int_{0}^{T_{\mathrm{R}}}|A(\tilde{z}, t)|^{4} \mathrm{~d} t}{\int_{0}^{T_{\mathrm{R}}}|A(\tilde{z}, t)|^{2} \mathrm{~d} t}, \\
& \Omega_{\mathrm{RMS}}(\tilde{z})=\sqrt{\frac{\int_{0}^{T_{\mathrm{R}}}\left|A_{t}(\tilde{z}, t)\right|^{2} \mathrm{~d} t}{\int_{0}^{T_{\mathrm{R}}}|A(\tilde{z}, t)|^{2} \mathrm{~d} t}}
\end{aligned}
$$

describing the RMS pulse width $T_{\mathrm{RMS}}$, the pulse power $P_{\mathrm{RMS}}$, the phase modulation parameter $C_{\mathrm{RMS}}$, and the spectral width $\Omega_{\text {RMS }}$. By solving Eqn (41) for RMS quantities assuming the parabolic phase of an optical signal [113], we obtain [65]

$$
\begin{aligned}
\frac{\mathrm{d} T_{\mathrm{RMS}}}{\mathrm{d} \tilde{z}} & =4 d(\tilde{z}) \frac{C_{\mathrm{RMS}}}{T_{\mathrm{RMS}}}-v g \frac{1}{T_{\mathrm{RMS}}^{2}}\left(I_{1}-1-T_{\mathrm{RMS}}^{2} \Omega_{\mathrm{RMS}}^{2}\right), \\
\frac{\mathrm{d} C_{\mathrm{RMS}}}{\mathrm{d} \tilde{z}} & =d(\tilde{z}) \Omega_{\mathrm{RMS}}^{2}-\frac{\varepsilon}{4} P_{\mathrm{RMS}}+v g\left(I_{2}+2 C_{\mathrm{RMS}} \Omega_{\mathrm{RMS}}^{2}\right), \\
\frac{\mathrm{d} P_{\mathrm{RMS}}}{\mathrm{d} \tilde{z}} & =-4 d(\tilde{z}) \frac{C_{\mathrm{RMS}} P_{\mathrm{RMS}}}{T_{\mathrm{RMS}}^{2}}+2\left(g-l_{0}\right) P_{\mathrm{RMS}}+ \\
& +2 v g\left(I_{3}+\Omega_{\mathrm{RMS}}^{2} P_{\mathrm{RMS}}\right), \\
\frac{\mathrm{d} \Omega_{\mathrm{RMS}}}{\mathrm{d} \tilde{z}} & =-2 \varepsilon \frac{C_{\mathrm{RMS}} P_{\mathrm{RMS}}}{T_{\mathrm{RMS}}^{2}}+2 v g\left(-I_{4}+\Omega_{\mathrm{RMS}}^{4}\right),
\end{aligned}
$$

where

$$
\begin{aligned}
& I_{1}=\int_{0}^{T_{\mathrm{R}}} \frac{t^{2}\left|A_{t}(\tilde{z}, t)\right|^{2}}{E} \mathrm{~d} t, \\
& I_{2}=-\mathrm{i} \int_{0}^{T_{\mathrm{R}}} \frac{t}{2 E}\left[A_{t}(\tilde{z}, t) A_{t t}^{*}(\tilde{z}, t)-A_{t}^{*}(\tilde{z}, t) A_{t t}(\tilde{z}, t)\right] \mathrm{d} t, \\
& I_{3}=\int_{0}^{T_{\mathrm{R}}} \frac{|A(\tilde{z}, t)|^{2}}{E}\left[A(\tilde{z}, t) A_{t t}^{*}(\tilde{z}, t)+A^{*}(\tilde{z}, t) A_{t t}(\tilde{z}, t)\right] \mathrm{d} t, \\
& I_{4}=\int_{0}^{T_{\mathrm{R}}} \frac{\left|A_{t t}(\tilde{z}, t)\right|^{2}}{E} \mathrm{~d} t, \\
& E=\int_{0}^{T_{\mathrm{R}}}|A(\tilde{z}, t)|^{2} \mathrm{~d} t .
\end{aligned}
$$

Dissipative terms in Eqn (41) give rise to moment integrals $I_{1}, \ldots, I_{4}$ in the system describing the dynamics of RMS characteristics, which makes it unclosed. However, the system can be simplified assuming that the optical pulse has a special shape. This allows calculating all RMS integrals, thereby closing the system of ODEs. In general, any approximation of the optical pulse can be used, for example, a hyperbolic secant of a Gaussian or parabolic profile. We consider a chirped Gaussian pulse of the form

$$
A(\tilde{z}, t)=\sqrt{P(\tilde{z})} \exp \left[-\frac{t^{2}}{2 \tau_{p}^{2}(\tilde{z})}(1-\mathrm{i} C(\tilde{z}))+\mathrm{i} \varphi(\tilde{z})\right] .
$$

In this case, the local and RMS characteristics of the signal are related by the expressions $T_{\mathrm{RMS}}=\tau_{p} / \sqrt{2}, C_{\mathrm{RMS}}=C / 4$, $P_{\mathrm{RMS}}=P / \sqrt{2}$, and $\Omega_{\mathrm{RMS}}^{2}=\left(1+C^{2}\right) /\left(2 \tau_{p}{ }^{2}\right)$.

The description of the dynamics of the central part of a dissipative soliton in terms of its key characteristics (duration 

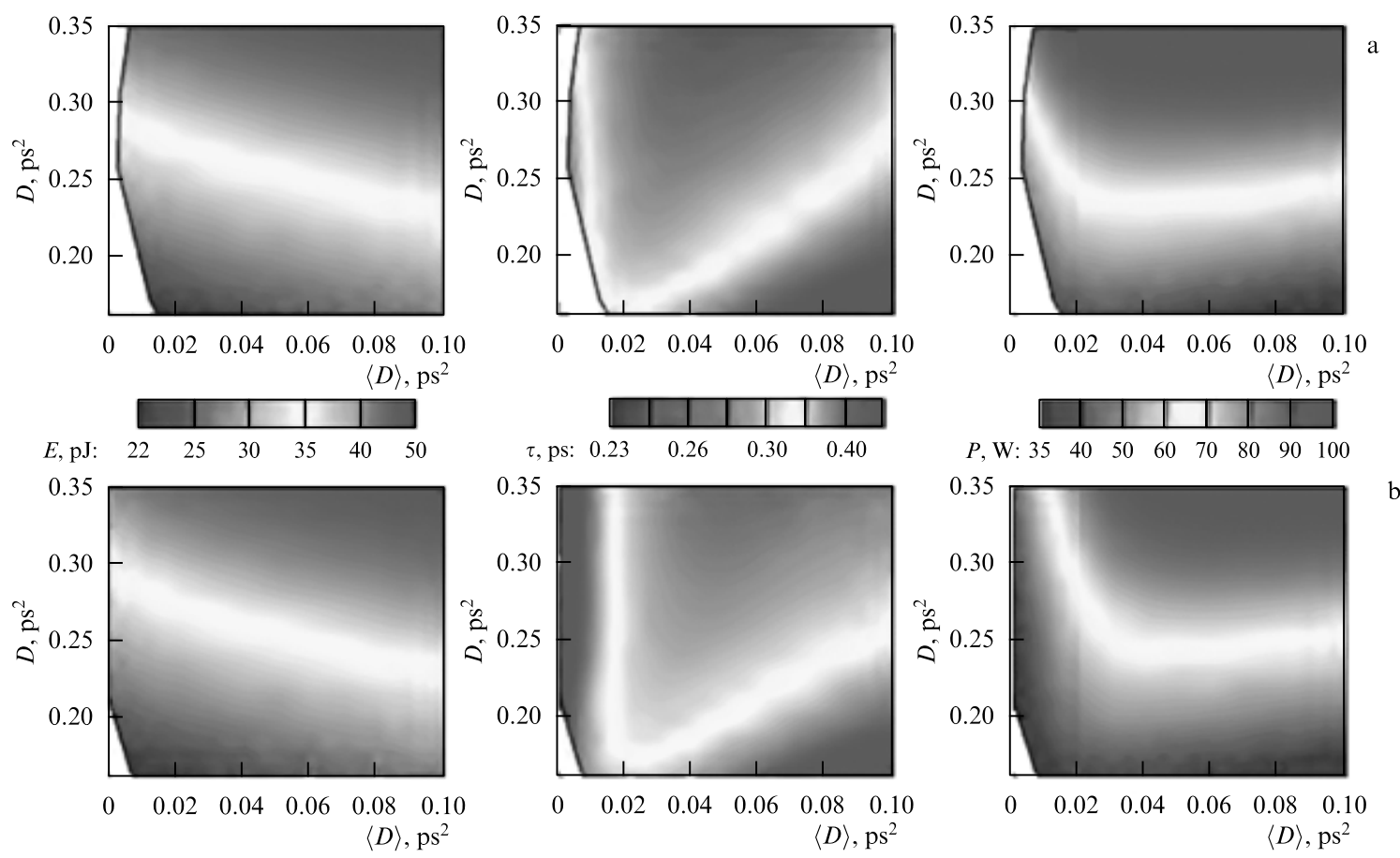

Figure 8. (Color online.) Level lines of the pulse energy $E$, the pulse width $\tau_{p}$, and the peak power $P$ obtained (a) by complete simulations and (b) by using the ODE system in the $(\langle D\rangle, D)$ plane for anomalous regimes.

$\tau_{p}$, peak power $P$, and chirp $C$ ) can then be reduced to a boundary value problem for the periodic solutions of the system of nonlinear ODEs [65, 108]:

$$
\begin{aligned}
& \frac{\mathrm{d} \tau_{p}}{\mathrm{~d} \tilde{z}}=2 d(\tilde{z}) \frac{C}{\tau_{p}}-v g \frac{1}{\tau_{p}}\left(C^{2}-1\right), \\
& \frac{\mathrm{d} C}{\mathrm{~d} \tilde{z}}=(2 d(\tilde{z})-2 v g C) \frac{1+C^{2}}{\tau_{p}^{2}}-\frac{\varepsilon}{\sqrt{2}} P, \\
& \frac{\mathrm{d} P}{\mathrm{~d} \tilde{z}}=-2 d(\tilde{z}) \frac{C P}{\tau_{p}^{2}}+2\left(g-l_{0}\right) P-2 v g \frac{P}{\tau_{p}^{2}},
\end{aligned}
$$

where

$$
g=g\left(\tau_{p}, P\right)=\frac{g_{0}}{1+P \tau_{p} \sqrt{\pi} / \varepsilon_{0}},
$$

with periodic boundary conditions

$$
\tau_{p}(0)=\tau_{p}(1), \quad C(0)=C(1), \quad P(0)=P(1) .
$$

Numerical simulations based on a system of ODEs are compared in detail with complete NSE simulations in [114], where the propagation of an optical pulse was simulated in a laser cavity with a dispersion map consisting of a fiber segment with the second-order dispersion and a fiber segment with negative dispersion. The average dispersion of the cavity (hereafter, all the quantities are scaled) is $\langle D\rangle=$ $d^{-}+d^{+}$, where $d^{-}$and $d^{+}$are the dispersions of fiber segments with negative and positive dispersions. The dispersion variation depth is $D=d^{-}-d^{+}$. A chromatic dispersion profile of the form

$$
d(z)= \begin{cases}D+\langle D\rangle, & 0 \leqslant z<0.25 \\ -D+\langle D\rangle, & 0.25 \leqslant z<0.75 \\ D+\langle D\rangle, & 0.75 \leqslant z<1\end{cases}
$$

was considered. Good agreement was achieved between simulation results for anomalous dispersion regimes. Figure 8 presents a comparison of level lines for the pulse energy $E$, the pulse width $\tau_{p}$, and the peak power $P$ obtained in the NSE (Fig. 8a) and ODE (Fig. 8b) simulations in the $(\langle D\rangle, D)$ plane for anomalous dispersion regimes.

Thus, for anomalous dispersion regimes, the results obtained by the method presented here are in good agreement with the results of NSE simulations. However, the time and especially the spectral characteristics of pulses are inconsistent with ODE simulations for normal dispersion regimes. Nevertheless, even in these cases, a simplified system of equations predicts the pulse energy sufficiently well.

\section{Dissipative Maxwell-Bloch solitons}

In the case of small variations in the characteristics of a pulse and medium for one round-trip transit of light in a cavity and comparatively short relaxation times of the active (amplifier) and passive (absorber) media, the formation dynamics of dissipative solitons can be described by Eqns (8) and (11) corresponding to the 'incoherent' self-mode matching (the pulse duration considerably exceeds the relaxation time of dipole momenta or the medium polarization). In [45, 46], it was additionally assumed that $\beta_{3}=0$ and $\gamma=0$ and time variables were normalized to $\tau_{\mathrm{sc}}=\sqrt{-\operatorname{Re} \beta_{2}}$ (anomalous dispersion). Then $\beta_{2}=-\left(1-\mathrm{i} d_{2}\right) \tau_{\mathrm{sc}}^{2}$, where $d_{2}=\tau_{\mathrm{sc}}^{-2} \operatorname{Im} \beta_{2}>0$ characterizes the width of the spectral filtration contour. Frequency detunings were assumed small compared to the width of the homogeneous amplification and absorption bands.

Analysis and numerical calculations confirm the existence of solutions of Eqns (8) and (11) in the form of stable localized structures. A necessary condition for the existence of dissipative solitons is the stability of the nonlasing regime. 

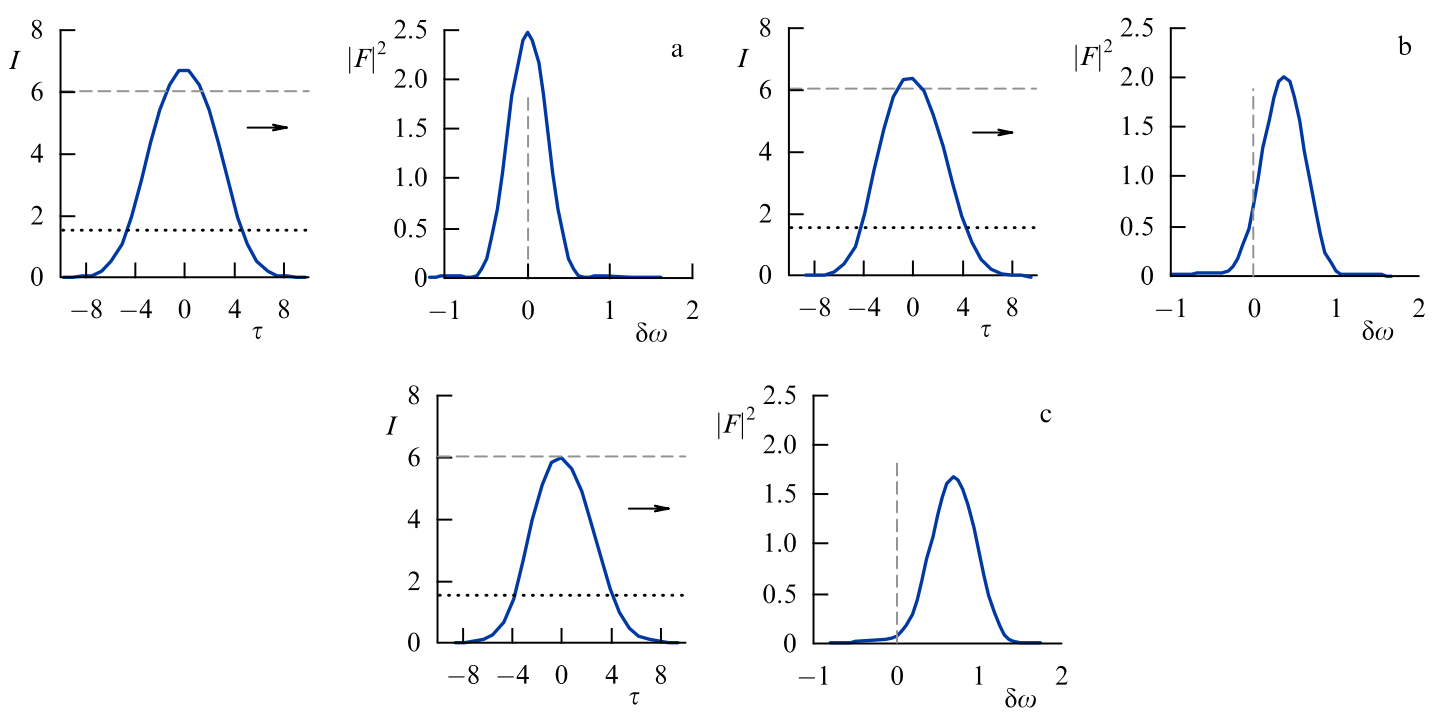

Figure 9. Intensity profiles $I(\tau)$ and the spectrum $|F(\delta \omega)|^{2}$ of stable solitons in a fiber with a rapid relaxation of the population difference (a) $\tau_{\mathrm{a}}=\tau_{\mathrm{p}}=0$, $d_{2}=0.1$; (b) $\tau_{\mathrm{a}}=\tau_{\mathrm{p}}=1, d_{2}=0.1$; (c) $\tau_{\mathrm{a}}=\tau_{\mathrm{p}}=1, d_{2}=0.05$. Pulse velocities in Figs $9 \mathrm{~b}$, c are smaller than the pulse velocity in an inertialess soliton (Fig. 9a); the frequency shift is positive. Frequency detuning in the figure is normalized to the time scale, $\delta \omega=\left(\omega-\omega_{21}^{\mathrm{a}}\right) \tau_{\mathrm{sc}}$.

In this case, weak initial perturbations resolve with time (resulting in efficient noise suppression in the soliton regime). But if the saturation intensity for the absorber is smaller than that for the amplifier, the saturated amplification exceeds the saturated absorption with increasing intensity and a strong excitation of lasing can occur. As a result, bistability takes place (lasing is absent or present for the same parameters, depending on the initial conditions). A laser soliton can be qualitatively visualized as a moving lasing islet against a nonlasing background.

In the case of fast nonlinearity, a discrete set of single solitons (with different time profiles, both stationary and pulsed) and bound solitons exists. The finite amplification and absorption relaxation times induce an asymmetry of the soliton profile and considerably affect the type of the interaction with each other. Numerical calculations show the existence of stationary solutions of Eqns (8) and (11) in a broad range of characteristic relative relaxation times $\tau_{\mathrm{a}}=$ $\tau_{\mathrm{p}}=0.1-10$, where $\tau_{\mathrm{a}, \mathrm{p}}=T_{1}^{(\mathrm{a}, \mathrm{p})} / \tau_{\mathrm{sc}}$. In the distributed loss approximation, the pulse shape is stabilized, and the pulse moves at a constant velocity different from the group velocity of light. In this case, the phase profile has a characteristic linear slope toward the soliton motion direction. For fixed characteristics of the medium, unique stationary values of the soliton velocity and frequency shift exist. If relaxation times are sufficiently small $\left(\tau_{\mathrm{a}} \approx \tau_{\mathrm{p}} \approx 1-3\right)$, the shape of a stationary soliton in a fiber weakly differs from that of a laser soliton with inertialess media $\left(\tau_{\mathrm{a}}=\tau_{\mathrm{p}}=0\right)$, the soliton velocity being smaller than the group velocity ('slow' soliton). Moreover, if the spectral filtration is absent $\left(d_{2}=0\right.$ and the medium is inertialess), the pulse is continuously slowed down, such that the stationary soliton does not exist (also see [115]). For a sufficiently small $d_{2} \approx 0.02$, the velocity shift is so large that it leads to a redistribution of the amplification and absorption balance sufficient for violating the soliton existence condition. Under these conditions, the soliton disperses and the nonlasing state sets in. For $d_{2} \approx 0.2-0.5$, the soliton velocity shift is small and the soliton is stable. Large filtration parameters break the balance between soliton losses and amplification even in inertialess media.

Figure 9 shows the stationary soliton intensity distributions and soliton spectra for different values of the parameter $d_{2}$ in a medium with fast nonlinearity $\left(\tau_{\mathrm{a}}=\tau_{\mathrm{p}}=0-1\right)$. We can see that as the relaxation time increases, the peak intensity decreases and the spectrum shifts to the blue side.

Figure 10 shows similar characteristics for 'fast' solitons in a fiber with a sufficiently inertial nonlinearity (relaxation times $\tau_{\mathrm{a}}=\tau_{\mathrm{p}}=10$ ). In this case, both the properties and the formation kinetics of such states qualitatively change compared to solitons in an inertialess medium. First of all, the soliton velocity increases and the velocity shift with respect to the group velocity is positive. The maximum intensity is two
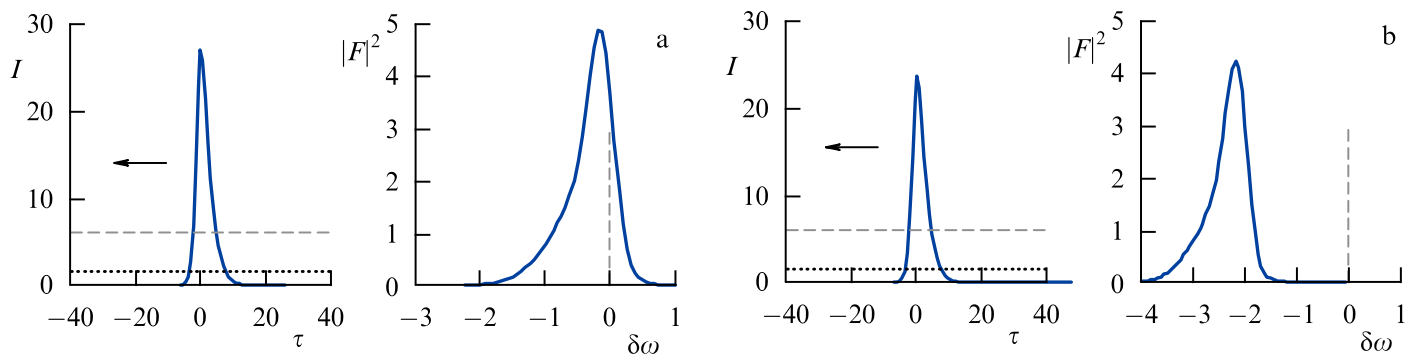

Figure 10. Intensity profiles and the spectrum of stable solitons in a fiber with slow relaxation of the population difference: $\tau_{\mathrm{a}}=\tau_{\mathrm{p}}=10,(\mathrm{a}) d_{2}=0.1$, (b) 0.01 . The pulse velocity is greater here than in the case of an inertialess soliton (Fig. 9a); the frequency shift is negative. 
to three times greater than the intensity of the homogeneous stationary state. The pulse duration in a medium with strong relaxation is approximately the same as in inetrialess media, but the pulse intensity at the periphery decreases more slowly and a relaxation 'tail' exists. The velocity of such a pulse is still stabilized at nonzero values of the parameter $d_{2}$ : the smaller the parameter is, the larger the positive velocity shift.

The soliton for $\tau_{\mathrm{p}}=0$ and $\tau_{\mathrm{a}}=1$ is stable in the interval $d_{2} \approx 0.3-0.6$. It loses its stability for $d_{2}>0.7$, dispersing with the establishment of a nonlasing state. However, for $d_{2}<0.2$, the stability loss scenario is different: the soliton width monotonically increases, while its intensity tends to the intensity of stable uniform lasing.

\section{Dissipative self-induced transparency solitons}

Progress in laser physics stimulates the development of techniques for generating shorter and shorter laser pulses, down to subfemtosecond (attosecond) pulses. The efficient generation of such pulses would allow time-resolved studies of the electron motion in atoms, molecules, and solids, the creation of compact electron and ion accelerators, and the generation of extremely short X-ray pulses for biological, medical, and technological applications. Modern approaches are based on the summation of radiation of multiple optical harmonics and the action of super-strong optical fields on thin targets, the efficiency of such approaches being restricted by the principal multi-stage type of physical processes involved.

The generation of extremely short pulses with a duration comparable to the inverse central radiation frequency directly in a laser can provide potentially high efficiency. To date, this method has been assumed to be fundamentally impossible, because the pulse duration is limited by the inverse width of the laser gain band [116]. This circumstance has led to the search for laser media with the widest gain band. A Ti:sapphire laser has the broadest gain band among known lasers. However, it is impossible to generate pulses shorter than two optical cycles even in this laser [117-119]. But this restriction can be eliminated in the extremal nonlinear optics regime when the gain band broadening caused by the pulse itself is so large that its width becomes comparable to the laser transition frequency.

This can be explained in two similar ways. First, we consider the example of a single-mode fiber with saturable amplification and absorption in the regime close to self- induced transparency $[120,121]$. In the absence of dissipative factors (amplification and absorption), self-induced transparency solitons form a family with a continuous spectrum of widths (and of the corresponding peak intensities). The introduction of linear amplification leads to successive replacement of solitons in the family with more and more narrow solitons during pulse propagation. Such a selfsharpening of initially standard (femtosecond) pulses is restricted by nonlinear amplification and absorption, resulting in the generation of ultrashort dissipative solitons with a duration of the order of the inverse laser transition frequency, i.e., a few femtoseconds or even subfemtoseconds. We note that ultrashort pulses are similar to $\pi$ pulses with respect to the absorber and to $2 \pi$ pulses with respect to the amplifier (with the subsequent slower kinetics determined by relaxation parameters). This results in an efficient energy extraction in the amplifier and the suppression of losses in the absorber. Second, the spectral band broadening effect caused by intense laser radiation is also known for comparatively long pulses in lasers with saturable absorbers, as is explained in [50], where the coherent mode matching technique was proposed and described theoretically. This regime was recently demonstrated experimentally in [122] for a dye laser with a molecular iodine absorption cell. This approach for ultrashort laser pulses has not been experimentally studied so far, and we present only some computer simulations of these regimes below. Details of the analysis are presented in [123-133].

We note that the dynamics of ultrashort pulses in long enough cavities, in which the round-trip time of light exceeds the relaxation times in the medium, and in fibers with saturable amplification and absorption do not fundamentally differ. In both cases, the radiation propagation is described by Eqn (1), i.e., without using the slowly varying envelope and unidirectional propagation approximations. The matter equations for the amplifier and absorber correspond to density matrix equations (4) and (5), again without using the slowly varying amplitude approximation. We note that in a number of cases it is necessary to pass from the two-level to the more complete three-level energy level diagram for a fiber system (Fig. 11). The frequency dispersion of the reflection coefficient of mirrors can be ignored in the simplest case.

The type of interaction of an ultrashort pulse with a medium is shown in Fig. 11. For active centers (amplifier), the pulse rapidly changes the population-difference sign,
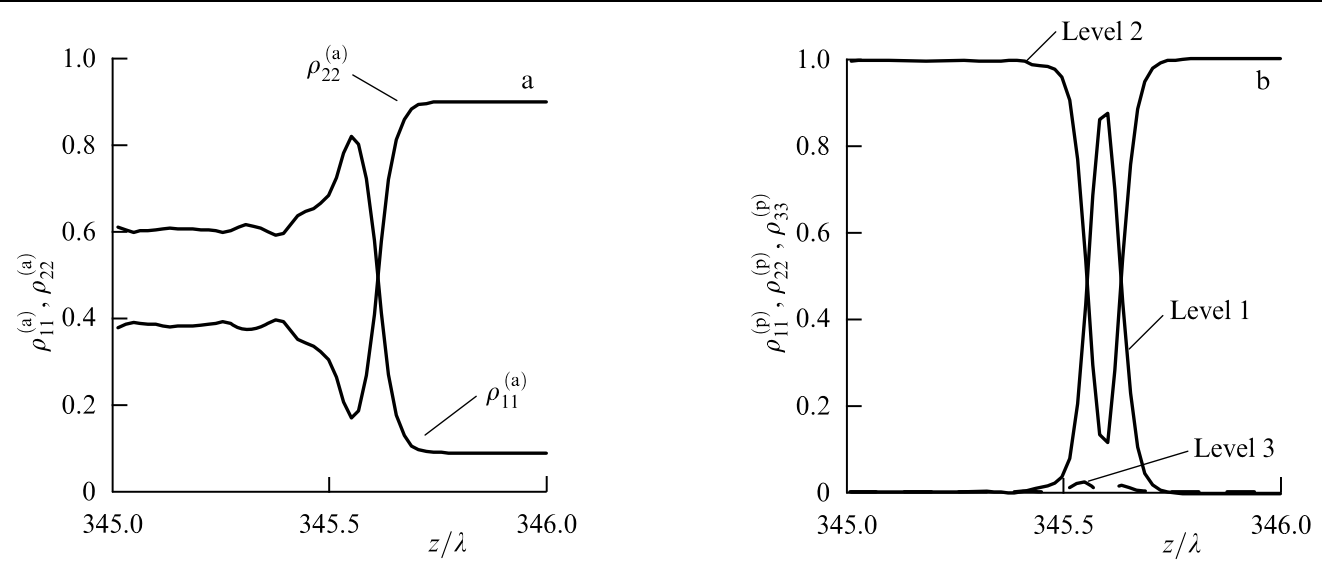

Figure 11. Evolution of populations of working levels 1 and 2 of (a) active and (b) 1,2, 3 passive centers during the propagation of a self-induced transparency pulse through them. 
acting as a $\pi$ pulse. This is followed by a long stage of recovering the initial level populations, determined by the relaxation time. For passive centers whose concentration greatly exceeds that of active centers, the level populations are practically recovered after the pulse propagation. Therefore, for them this is a $2 \pi$ pulse. The formation dynamics of ultrashort pulses are similar in the case of inhomogeneous broadening.

To generate dissipative self-induced transparency solitons in active silica crystals and fiber lasers, a number of exactly conditions must be satisfied. It is important that the resonance nonlinearity dominate, while a silica matrix behave as weakly nonlinear at such intensity levels. This is achievable, for example, if active centers are quantum dots in which transition dipole moments greatly exceed those in atomic systems. The factor restricting the duration of an ultrashort pulse is the IR and UV absorption in the matrix. Accordingly, the pulse spectrum (coherent supercontinuum) covers almost the entire transparency region of the matrix, being restricted to its absorption bands.

\section{Examples of dissipative solitons in fiber lasers}

\subsection{Dissipative solitons in long fiber lasers}

The cavity length is an important parameter of a fiber laser system determining optical pulse characteristics [134]. The pulse energy in fiber lasers can be considerably increased by increasing the cavity length $[52,53]$. Mode locking has been demonstrated in a laser $25 \mathrm{~km}$ in length [135], and the maximum pulse energy equal to $4 \mu \mathrm{J}$ was achieved in very long mode-locked fiber lasers [136]. A great number of recent theoretical and experimental studies have been devoted to high-energy pulsed fiber lasers with cavities a few kilometers in length without using conventional techniques such as Qswitching or open cavities (see, e.g., [52-54, 137-139]). Fiber lasers allow the use of many nonlinear mechanisms for radiation generation and shaping $[48,65,82,103,105,108$, $112,140-142]$. We note that nonlinear effects make the numerical simulation of fiber lasers a quite challenging problem. The numerical simulation of fiber lasers with very long cavities is complicated due to technological problems such as time-consuming calculations and the necessity of using considerable computational resources. Recent numerical simulations were used to analyze the properties of long, passively mode-locked fiber lasers based on nonlinear optical rotation with the cavity lengths from $100 \mathrm{~m}[137,138]$ to $8 \mathrm{~km}$ [53].

Figure 12a shows a schematic of a fiber laser considered in numerical simulations. A ring cavity consists of an active erbium fiber $2 \mathrm{~m}$ in length, a passive fiber with a variable length, a saturable absorber, and a coupler. A pulse in cavities with anomalous dispersion usually exhibits soliton instabilities [108]. It is known that the influence of nonlinear effects resulting in pulse destruction increases upon increasing the length of a cavity with anomalous mean dispersion. Singlepulse regimes were obtained only for cavity lengths not greater than $10 \mathrm{~m}$ using a standard SMF-28 single-mode fiber. The cumulative dispersion of the cavity in this case was $-0.0742 \mathrm{ps}^{-2}$. To avoid the appearance of soliton instabilities, a long fiber laser was simulated using an OFS-980 fiber with positive dispersion.

Numerical experiments were performed using a pointwise approach with each element described by the corre-
Table 1. Parameters of the fiber laser.

\begin{tabular}{|l|l|l|}
\hline \multicolumn{1}{|c|}{ Element } & \multicolumn{1}{|c|}{ Parameter } & \multicolumn{1}{c|}{ Value } \\
\hline $\begin{array}{l}\text { Active erbium- } \\
\text { doped fiber } \\
{[140]}\end{array}$ & $\begin{array}{l}\text { Length } L_{\mathrm{AF}} \\
\text { Second-order dispersion } \beta_{2}\end{array}$ & $2 \mathrm{~m}$ \\
& $\begin{array}{l}\text { Third-order dispersion } \beta_{3} \\
\text { Nonlinearity parameter } \gamma\end{array}$ & $168 \mathrm{fs}^{2} \mathrm{~mm}^{-1}$ \\
& Gain spectrum width $\Lambda_{\mathrm{G}}$ & $0.00932 \mathrm{~W}^{-1} \mathrm{~m}^{-1}$ \\
& Gain $G_{0}$ & $50 \mathrm{~nm}$ \\
& Saturation power $P_{\mathrm{sat} \mathrm{G}}$ & $5.4 \mathrm{~dB} \mathrm{~m}$ \\
& $20 \mathrm{~mW}^{-1}$ \\
\hline $\begin{array}{l}\text { Passive } \\
\text { OFS-980 fiber } \\
{[140]}\end{array}$ & Second-order dispersion $\beta_{2}$ & $4.5 \mathrm{fs}^{2} \mathrm{~mm}^{-1}$ \\
& Third-order dispersion $\beta_{3}$ & $109 \mathrm{fs}^{3} \mathrm{~mm}^{-1}$ \\
Nonlinearity parameter $\gamma$ & $0.0021 \mathrm{~W}^{-1} \mathrm{~m}^{-1}$ \\
& Fiber losses $\alpha$ & $0.2 \mathrm{~dB} \mathrm{~km}^{-1}$ \\
\hline Saturable ab- & Modulation depth $q_{0}$ & $10 \%$ \\
sorber $[108]$ & Saturation power $P_{\text {sat }}$ & $3.69 \mathrm{~W}$ \\
\hline Coupler & Output losses $R_{\text {out }}$ & Varying around \\
& & $90 \%$ \\
\hline
\end{tabular}

sponding individual mathematical model. The details of simulations are presented in [143]. The parameters of the laser system elements are presented in Table 1 . The laser wavelength was $\lambda_{0}=1550 \mathrm{~nm}$. The round trip time of light in this cavity is $T_{\mathrm{R}}=n_{0} L / c$, where $L=L_{\mathrm{AF}}+L_{\mathrm{PF}}$ is the total cavity length and $L_{\mathrm{PF}}$ is the passive fiber length; the refractive index of the fiber core is $n_{0} \approx 1.5$.

The study demonstrated the importance of the initial field distribution in simulations of pulse generation in long-cavity fiber lasers. A smooth initial small-amplitude distribution (for example, a Gaussian pulse) is commonly used for simulating mode-locked fiber lasers. This simplification is based on the assumption that the asymptotic state is generated after many round trips in the cavity and is independent of any features of the initial noise. As a rule, the initial conditions do not play a considerable role in the formation of the final asymptotic state in a nonlinear dissipative laser system. However, as the cavity length is increased, the resulting states can be different for a smooth initial field distribution and the noise distribution, as we show below. The initial distributions used in calculations were white noise and a small-amplitude Gaussian pulse. The number of nodes in time was $2^{14}$ and the number of nodes along the evolution variable was 4000 per round trip of light in the cavity.

It was found that pulsed regimes established in the laser depend on the initial conditions. For example, single-pulse regimes obtained from a smooth initial distribution do not necessarily coincide with regimes obtained from the noisy initial distribution with the same mean power.

For relatively short cavities (up to $32 \mathrm{~m}$ ), the same established single-pulse regimes can be obtained from considerably different self-starts. To stabilize the pulse energy for a cavity $32 \mathrm{~m}$ in length, more than 4000 round trips in the cavity are required. To achieve a stable state in terms of the energy evolution in shorter cavities, about 1000 round trips were required. However, for cavity lengths $\geqslant 42 \mathrm{~m}$, stable pulsed regimes cannot be achieved even after 129,000 round trips in the cavity. Calculations showed that the required number of round trips in a cavity more than $20 \mathrm{~m}$ in length increases exponentially.

The black line in Fig. 12b shows the resulting pulse shape and its spectrum for a cavity $32 \mathrm{~m}$ in length. The inset in Fig. 12b shows that the pulse spectrum has the shape typical for strongly chirped pulses with sharply decaying edges [112]. 

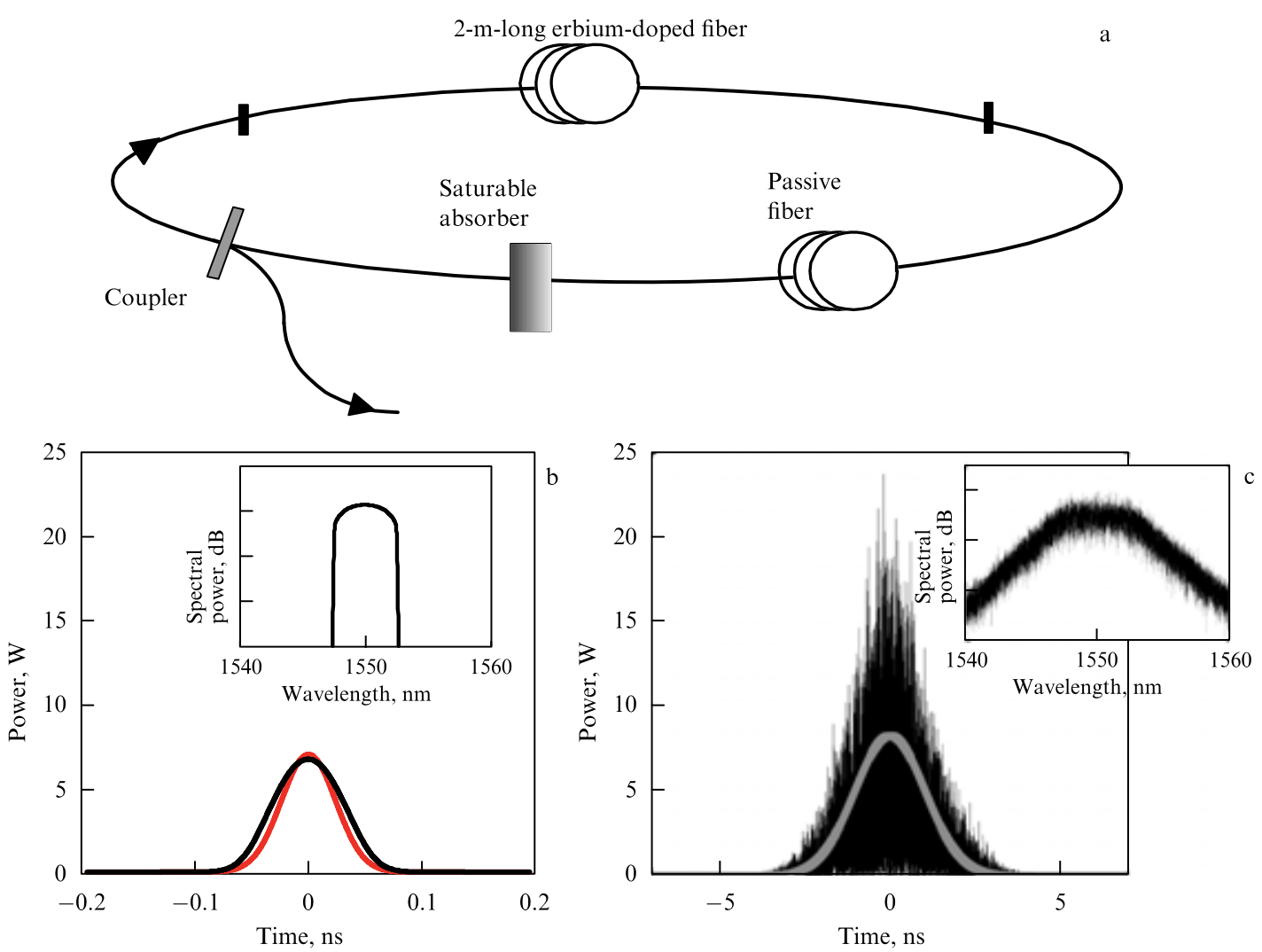

Figure 12. (Color online.) (a) Schematic of a dissipative soliton fiber laser. (b, c) Pulse shapes. Black curves are pulses generated from white noise as the initial distribution, the red curve is the approximation by a Gaussian pulse constructed by RMS characteristics for (b) $32 \mathrm{~m}$ long and (c) $2002 \mathrm{~m}$ long cavities. Insets show the corresponding averaged pulse spectra obtained from white noise.

The black line in Fig. 12c illustrates the typical pulse shape after 2000 round trips of light in a cavity $2002 \mathrm{~m}$ in length. We failed to achieve a smooth pulse shape in simulations even after 20,000 round trips of light in the cavity. The typical resulting pulse shape is an envelope with a width of several nanoseconds and with strong noisy oscillations in the envelope. The noisy component can be compared with pulsed collapsing regimes studied in [141, 142]. We assume that the pulse is stable when the relative change $\varepsilon=$ $\left|E_{i}-E_{i+1}\right| / E_{i}$ in the pulse energy $\varepsilon$ does not exceed $10^{-3}$. The resulting pulse energy was $0.5 \mathrm{~nJ}$ and $16 \mathrm{~nJ}$ for the respective cavity lengths of 32 and $2002 \mathrm{~m}$.

The insets in Figs 12b, c show the averaged spectral pulse shapes obtained from the initial white noise. In the case of a cavity $2002 \mathrm{~m}$ in length, the spectrum was averaged over the last 10 round trips in the cavity. We can see from the plot that the different amplifications for cavities 32 and $2002 \mathrm{~m}$ in length can be explained by different spectral characteristics.

For comparison, the red line in Fig. 12b shows a Gaussian pulse having the same RMS characteristics as the obtained pulses and specified in the standard way: for a Gaussian pulse described by the expression $\sqrt{P} \exp \left(-t^{2} / \tau^{2}\right)$, the corresponding RMS characteristics are $P=\sqrt{2} P_{\mathrm{RMS}}$ and $\tau^{2}=4 T_{\mathrm{RMS}}^{2}$. Here, the FRMS characteristics of the pulse are defined in the standard way as

$$
P_{\mathrm{RMS}}=\frac{\int_{0}^{T_{\mathrm{R}}}|A|^{4} \mathrm{~d} t}{E}, \quad T_{\mathrm{RMS}}^{2}=\frac{\int_{0}^{T_{\mathrm{R}}} t^{2}|A|^{2} \mathrm{~d} t}{E},
$$

where $P_{\mathrm{RMS}}$ and $T_{\mathrm{RMS}}$ are the RMS pulse power and width, and $E=\int_{0}^{T_{\mathrm{R}}}|A|^{2} \mathrm{~d} t$.
Figure 13 shows the intracavity dynamics and RMS width and power for a pulse obtained from the initial white noise for different cavity lengths. It can be seen that the RMS pulse widths change differently in short and long cavities. The RMS pulse width in the long cavity weakly decreases in the amplifying medium. Nevertheless, the relative change in the RMS pulse widths is negligible in both cases.

The pulse is amplified in the active fiber and the behavior of the RMS width during the pulse propagation in the active fiber depends on the total cavity length. The passive fiber broadens the pulse and slightly reduces its power; the saturable absorber reduces the RMS pulse width and slightly reduces the RMS pulse power. The coupler also reduces the pulse power. The difference between the dynamics of the RMS pulse widths for the two regimes is due to the definition of the RMS pulse characteristics and can be explained by the greater increase in the pulse energy in the long cavity that in the cavity $32 \mathrm{~m}$ in length. We note that the pulse broadens in the active fiber in both long and short fibers. The zero-chirp point is absent in the laser cavity, which is typical for lasers with the everywhere. Normal dispersion [112]. The intracavity dynamics substantially change in long fiber lasers.

The pulse energy loss in a saturable absorber is determined not only by the modulation depth but also by the pulse energy to the saturation energy ratio. Thus, in fact, real losses in the saturable absorber are lower than $10 \%$ (real losses were $4 \%$ for cavities $32 \mathrm{~m}$ and $2 \mathrm{~km}$ in length). For this reason, the saturable absorber very weakly affects the pulse energy. For the larger modulation depth, the resulting pulse has the lower energy and shorter duration. The pulse energy decreases 


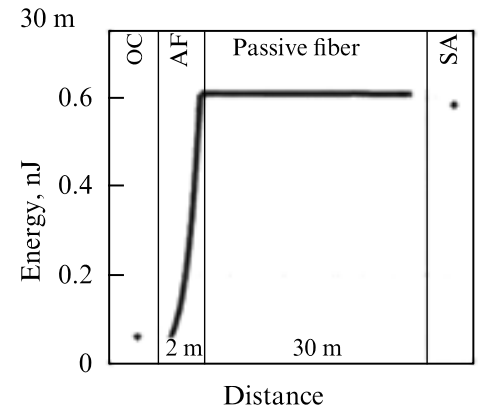

$2000 \mathrm{~m}$
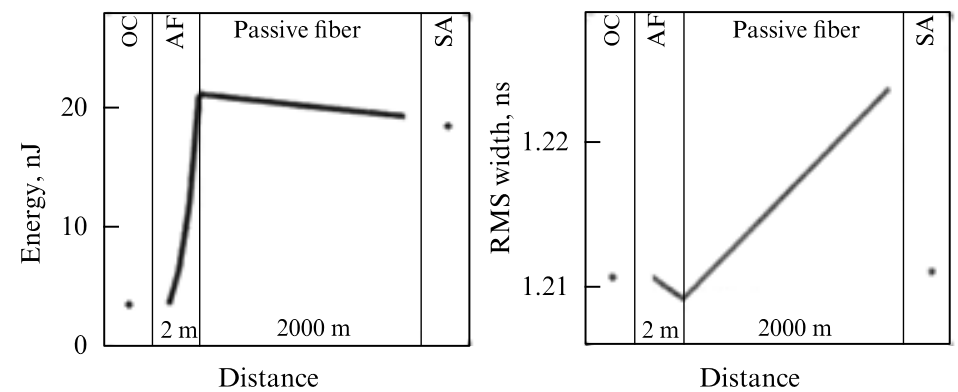
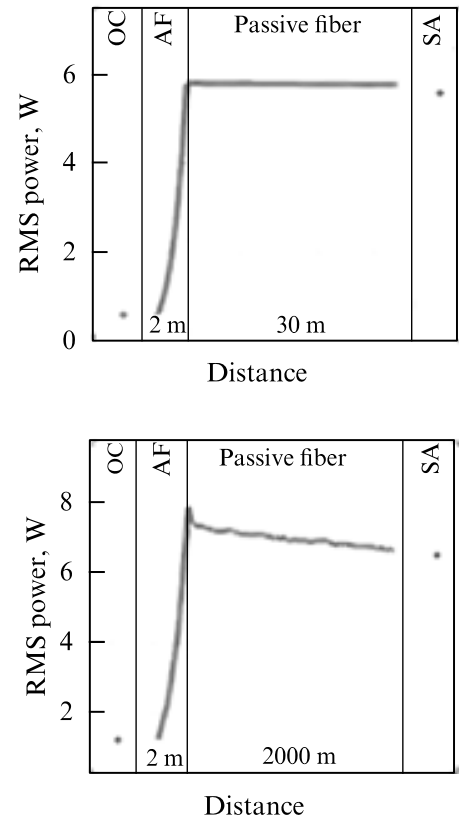

Figure 13. Intracavity energy dynamics and RMS characteristics of a pulse obtained from the initial Gaussian noise for different cavity lengths.

mainly due to its more greater compared to the decrease in its power. For the cavity $32 \mathrm{~m}$ in length, the pulse energy decreases from 0.58 to $0.54 \mathrm{~nJ}$ upon increasing the modulation depth from 10 to $13 \%$ (the saturation power did not change). In this case, real losses were $5 \%$. When the modulation depth parameter exceeded $15 \%$, lasing disappeared.

The influence of the cavity length on the pulse energy was numerically analyzed using the parameter $R_{\text {out }}$ (the output cavity losses) to control the energy balance by maintaining the constant gain $G_{0} L_{\mathrm{AF}}$. As a whole, an increase in the passive fiber length leads to an increase in the pulse energy and gain saturation. This is compensated by the control of losses at the cavity output and the increase in losses in the fiber [103]. In other words, total losses in the cavity $\left(R_{\mathrm{in}}+\alpha L_{\mathrm{PF}}\right)[\mathrm{dB}]$, where $R_{\text {in }}[\mathrm{dB}]=10 \ln \left(1-R_{\text {out }}\right) / \ln 10$ and $L_{\mathrm{PF}}$ is the passive fiber length, change with increasing the cavity length, but first of all due to the gain saturation and control of output lasses, whereas additional losses in the passive fiber play a minor role. Calculations show that the dependences of the cavity output energy on the fiber length are different for short and long cavities. The output energy depends linearly on the fiber length for short cavities. But its increase is saturated at large lengths due to a change in the ratio between losses and amplification. Calculations show that this dependence can be approximated by the curve

$$
E=a L \frac{G_{0} L_{\mathrm{AF}}-\left(R_{\mathrm{in}}+\alpha L_{\mathrm{PF}}\right)}{R_{\mathrm{in}}+\alpha L_{\mathrm{PF}}},
$$

where $a$ is a constant.

For cavity lengths above $2002 \mathrm{~m}$, no stable (defined above) single-modes were found.

\subsection{Dissipative solitons} in a SESAM mode-locked femtosecond fiber laser In this section, we consider the optimization of a SESAM mode-locked fiber laser.
Figure 14a shows a diagram of a fiber laser [144] used in numerical simulations of the successive propagation of a pulse in different elements of the laser [104]. The cavity consists of a coupler, a $1.5 \mathrm{~m}$ long passive fiber, a $0.5 \mathrm{~m}$ long active fiber, a pair of diffraction gratings providing anomalous dispersion with a very weak nonlinearity, and a SESAM (semiconductor saturable absorber mirror). The cumulative dispersion of passive and active fibers was $0.08 \mathrm{ps}^{2}$ per round trip in the cavity.

The details of numerical simulations are presented in [105]. The nonlinearity and dispersion coefficients of passive and active fibers were $\gamma=5 \mathrm{~W}^{-1} \mathrm{~km}^{-1}, \beta_{2}=20 \mathrm{ps}^{2} \mathrm{~km}^{-1}$, and $\beta_{3}=0.05 \mathrm{ps}^{3} \mathrm{~km}^{-1}$. The unsaturated SESAM losses were $4 \%$.

The region of parameters in which stable single-pulse and multipulse lasing was observed was determined from numerical calculations. Stable lasing regions and the difference between single-pulse and multipulse regimes were determined by monitoring the relative changes in the key characteristics of the signal during the last 200 round trips of light in the cavity until the establishment of a stable state. In general, lasing regimes were considered stable if relative changes in the pulse energy $\Delta E_{p}$ in the coupler did not exceed $10^{-3}$ and relative changes in the pulse width and its peak power satisfied the conditions $\Delta T_{\mathrm{FWHM}}<10^{-2}$ and $\Delta P_{p}<10^{-2}$. Thus, the accepted criterion allowed changes in pulse parameters within a percent.

Figures 14b, c show stable multipulse lasing regions in the (saturable-absorber recover time, saturation energy) and the (gain, cumulative dispersion) planes for a SESAM with the modulation depth $q_{0}=0.1$. The cumulative dispersion of the cavity was changed in experiments by changing the dispersion of a pair of diffraction gratings. Lines in Fig. 14b bound regions with different numbers of pulses in the cavity. The region bounded by the solid line corresponds to single-pulse lasing regimes, while the region bounded by the dashed line, to the two- and three-pulse regimes and regimes with the number of pulses in the cavity greater than two. We note that 

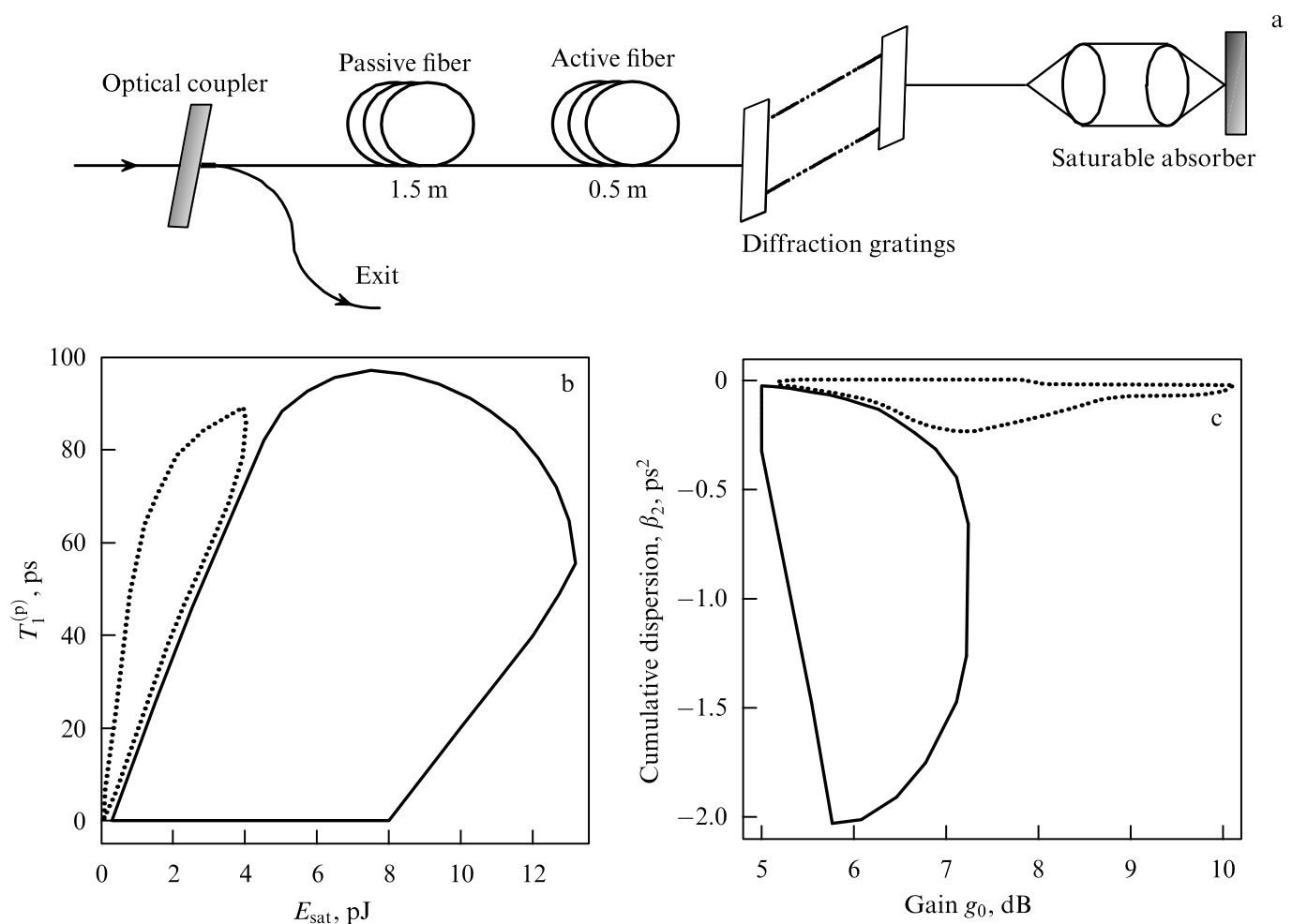

Figure 14. (a) Diagram of a mode-locked fiber laser. (b, c) Regions of stable multipulse lasing: the solid curve bounds the single-pulse lasing region, the dashed curve bounds two- and three-pulse regimes.

multipulse regimes are quite sensitive to the initial noise, and therefore two or more pulses can be generated in the same region depending on the initial noise structure. We can see from Fig. 14 that single-pulse lasing occurs in a quite broad range of parameters.

We note that the high saturation energy and the short recovery time lead to single-pulse lasing.

Figure 14c shows that single-pulse regimes exist for anomalous dispersion for the total gain in the range 5$7.5 \mathrm{~dB}$. The temporal shape and optical spectrum of such a stable pulse are presented in Fig. 15a for different initial noise realizations. The pulse spectrum has sidebands caused by periodic changes in soliton parameters during round trip of light in the cavity [145]. We note that the results are shown for 17 different initial noise realizations. It can be seen that the time and spectral shapes of a stable pulse are independent of the details of the initial noise distribution. Figure 15b shows the intracavity dynamics of the stable single-pulse regime in a fiber laser. We can see that the established pulses are dispersion-controlled solitons (see, e.g., [95, 145-148]) with the temporal and spectral shape periodically reproduced after each round trip in the cavity and pulse parameters are fixed by characteristics of the system. Key characteristics such as the pulse duration, energy, chirp, and spectral width practically coincide at the end of each round trip in the cavity.

We note that Fig. $15 \mathrm{~b}$ demonstrates considerable changes in the pulse parameters in the cavity. This should be taken into account for properly locating the coupler in the cavity. We can see from Fig. 15b that the pulse duration is minimal at the zero-chirp point immediately after the propagation of light through a dispersion compensator. The location of the coupler in front of or close to this point allows the generation of the shortest output pulse.

\subsection{Dissipative solitons in a nonlinear optical-rotation mode-locked laser}

The evolution of a signal in a mode-locked fiber laser based on nonlinear optical rotation was analyzed for a dissipative soliton ring laser, shown schematically in Fig. 16 [149].

The parameters of this laser are presented in Table 2 . The laser consists of a $15 \mathrm{~cm}$ long active single-mode fiber doped with $\mathrm{Yb}^{3+}$ at a high concentration, an $80 \mathrm{~cm}$ long passive fiber, and an isolator. Optical elements in the volume part of the laser include a quarter-wave plate (QWP), a half-wave plate (HWP), a polarizer and a $4 \%$ plane for controlling intracavity laser parameters, and a second QWP for obtaining a weak elliptic polarization at the input to the fiber part of the cavity, which is necessary for the nonlinear evolution of polarization. The laser wavelength was $1030 \mathrm{~nm}$.

Amathematicalmodelof thelaseris presented in Section 2.3. The details of numerical simulations are given in [153].

The system of coupled Ginzburg-Landau equations describes the propagation of light in the fiber part of the cavity. The polarizer in the volume optical part of the cavity transforms the elliptic polarization to linear. One of the

Table 2. Parameters of a fiber laser.

\begin{tabular}{|c|l|l|}
\hline Element & \multicolumn{1}{|c|}{ Parameter } & \multicolumn{1}{c|}{ Value } \\
\hline Active fiber & Length $L_{\mathrm{AF}}$ & $15 \mathrm{~cm}$ \\
& Second-order dispersion $\beta_{2}$ & $25 \mathrm{ps}^{2} \mathrm{~km}^{-1}$ \\
& Nonlinearity parameter $\gamma$ & $5 \mathrm{~W}^{-1} \mathrm{~km}^{-1}$ \\
& Gain bandwidth $\Lambda_{\mathrm{G}}$ & $20 \mathrm{~nm}$ \\
& Gain $g_{0}$ & $150-170 \mathrm{~dB}$ \\
& Saturation power $P_{\text {sat G }}$ & $17 \mathrm{~mW}$ \\
\hline \multirow{2}{*}{ Passive fiber } & Length & Variable \\
& Second-order dispersion $\beta_{2}$ & $-0.07323 \mathrm{ps}^{2} \mathrm{~m}^{-1}$ \\
& Nonlinearity parameter $\gamma$ & $5 \mathrm{~W}^{-1} \mathrm{~km}^{-1}$ \\
\hline
\end{tabular}



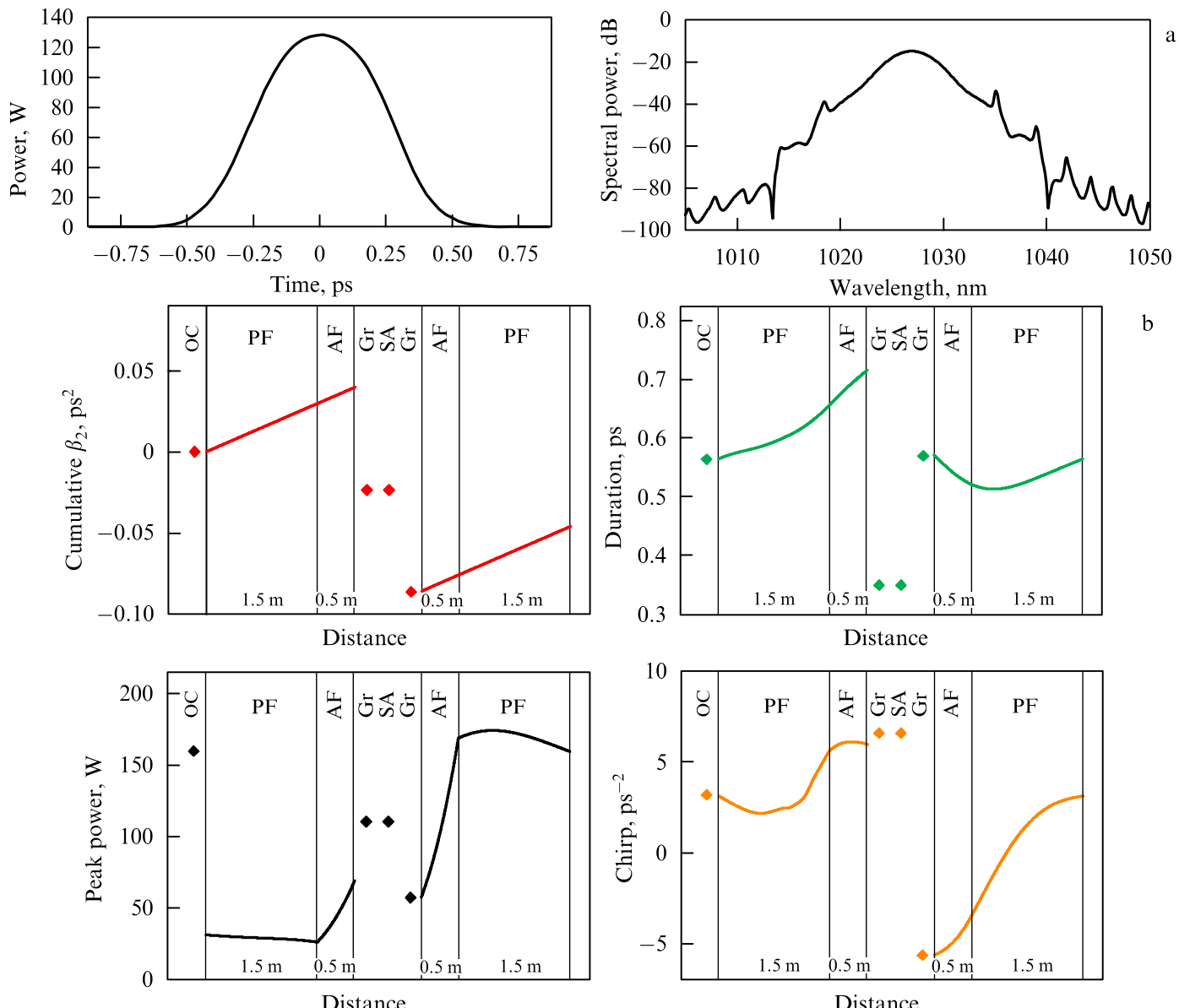

Figure 15. (a) Pulse shape and spectrum. (b) Evolution of the characteristics of the single-pulse intracavity regime. Calculations were performed for the parameters $g_{0}=5.5 \mathrm{~dB}, \beta_{2}=-0.046 \mathrm{ps}^{2}, \beta_{3}=5 \times 10^{-4} \mathrm{ps}^{3}, q_{0}=0.3, E_{\text {sat }}=0.5 \mathrm{pJ}$, and $\tau_{\text {sat }}=10 \mathrm{ps}$.
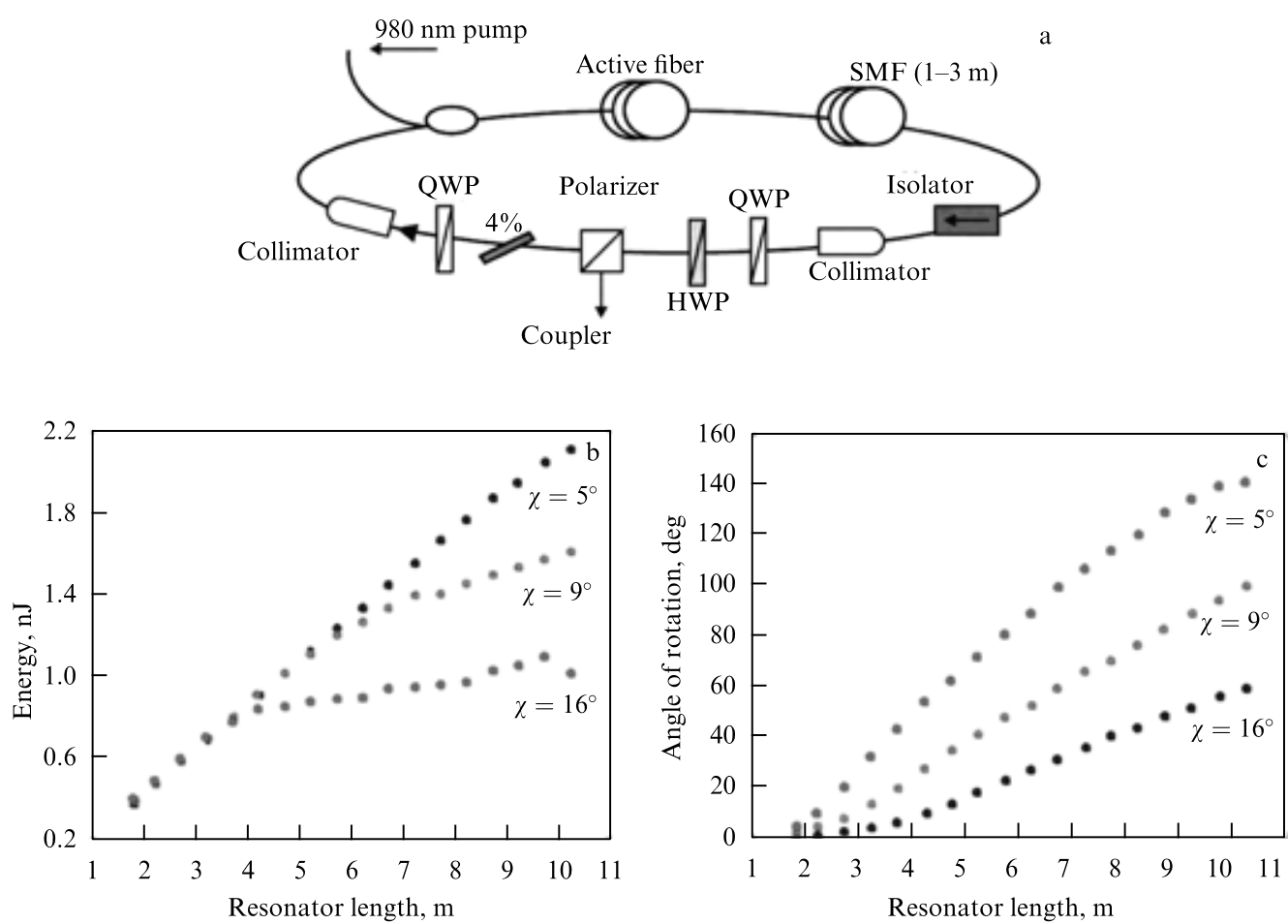

Figure 16. (a) Schematic of a passively mode-locked fiber laser based on nonlinear optical rotation. (b) Dependence of the maximum output pulse energy on the cavity length. The maximum pulse energy was obtained by varying the gain. (c) Dependences of the total angle of rotation of the polarization ellipse per round trip on the cavity length for $\chi=5^{\circ}, 9^{\circ}$, and $16^{\circ}$. 
polarized components passing through the polarizer escapes from the cavity via the coupler and does not participate in the subsequent pulse propagation, whereas the orthogonally polarized component modified in the QWP enters the fiber part of the cavity. By varying the angles of rotation of the QWP and HWP, we can control the polarization of light at the fiber input, in particular, its ellipticity and the angle of the polarization ellipse axis.

The numerical simulation was performed for different cavity lengths and different angles of rotation of the QWP and HWP. The output pulse power was optimized for each wavelength by rotating an HWP (this corresponds to a variation of the angle $\psi$ ), whereas the polarization ellipticity at the fiber output was fixed with the help of a QWP. The second QWP shown in Fig. 16a played only an auxiliary role in the experiments and is not presented in the numerical model.

Simulations were performed for three different angles of rotation of a QWP: $\chi=0.01 \pi, 0.03 \pi$, and $0.05 \pi$, corresponding to 5,9 , and $16^{\circ}$. The dependences of the maximum output pulse energy on the cavity length for three different input ellipticities determined by these angles are presented in Fig. 16b. The maximum pulse energy for each combination of the cavity length and the QWP rotation angle was achieved by varying the gain $g_{0}$. As a result, the maximum value of $g_{0}$ was chosen at which stable single-pulse lasing was achieved. If the gain taken for a certain set of parameters was too high, the generation of a dissipative soliton became unstable and mode locking became stochastic.

Calculations showed that the maximum output pulse energy linearly increases with the cavity length, but this dependence changes at some critical length (Fig. 16b). The critical length depends on the light ellipticity at the input to the fiber part. The higher the ellipticity is (i.e., the higher the angle $\chi$ of plate rotation), the lower the maximum pulse energy and the critical length above which the pulse energy dependence on the cavity length begins to deviate from the linear dependence. Hence, to increase the critical length and maximum pulse energy, it is necessary to couple the light with the lowest possible ellipticity to the fiber part. However, in reality, the ellipticity is restricted by the influence of random birefringence in the fiber, resulting in fluctuations during the nonlinear evolution of polarization. The interplay of these effects allows the maximum critical length to be determined.

The dependence of the total angle of rotation of the polarization ellipse per round trip of light on the length of the cavity is presented in Fig. 16c. We note that the maximum pulse energy is restricted by the accessible angle of rotation of the polarization ellipse per round trip in the cavity. Figure 16c shows the total angle of nonlinear rotation of the polarization ellipse caused by the nonlinear evolution of polarization as a function of the cavity length. For the critical cavity length, when the maximum pulse energy no longer increases with the fiber length, the rotation angle tends to $\pi / 2$, resulting in an ambiguity in the self-amplitude modulation. Thus, to obtain stable mode-locking due to the nonlinear evolution of polarization, the relation between the angles of rotation of polarization plates, the gain, and the fiber length should provide an angle of rotation of the polarization ellipse axis smaller than $\pi / 2$. For larger angles, the stability of strongly chirped lasing regimes is lost, resulting in the disappearance of stable single-pulse lasing and a transition to the multipulse and stochastic regimes.
Thus, the limit of the maximum pulse energy can be overcome by maintaining the angle of rotation of the polarization ellipse smaller than $\pi / 2$. To maintain the angle of rotation smaller than $\pi / 2$, it is necessary to increase the total cavity length by increasing the length of a polarizationpreserving fiber. At the same time, the length of a standard single-mode fiber should be fixed (or even shortened) for maintaining the angle of rotation of the polarization ellipse, formed by the nonlinear evolution of polarization, within $\pi / 2$. As a result, the nonlinear phase increment (and the corresponding dispersion phase increment) during pulse propagation in the polarization-preserving fiber leads to the formation of a strongly chirped soliton, whereas optical rotation in a standard single-mode fiber provides stable mode-locking.

\subsection{Raman dissipative solitons}

The generation of dissipative solitons (DSs) is one of the most efficient techniques for obtaining energy-scaled femtosecond pulses in mode-locked femtosecond lasers [48, 150]. For example, the authors of [151] demonstrated a new femtosecond fiber laser emitting high-energy pulses (up to $25 \mathrm{~nJ}$ ) by means of a decrease in the pulse repetition rate. They also discovered that the pulse energy was restricted at a level of a few tens of nanojoules due to the appearance of a stimulated Raman scattering (SRS) noise pulse. The SRS pulse forms with a stable complex in which the DS serves as a pump source for the pulse, whereas the pulse provides spectral and time filtration for the DS [152]. The mathematical simulation of a fiber laser in [152] showed that a stochastic SRS pulse is generated from the amplifier noise in each round trip in the cavity.

The possibility of generating an SRS pulse not from the amplifier noise but from a small-amplitude seed pulse was studied in [153]. The authors proposed introducing a new intracavity element, a delay line for the SRS pulse providing weak feedback for it in each round trip in the cavity. Because of dispersion in the fiber, the DS and SRS acquire different group velocities $v_{\mathrm{s}}$ and $v_{\mathrm{r}}$. The SRS pulse propagates in the fiber faster than the DS, and therefore it is amplified during a finite time equal to the DS duration. After propagation through the interaction region, the DS continues to propagate without energy losses, while the Stokes pulse begins to decay. As a result, the SRS pulse is shifted with respect to the pump pulse by approximately $50 \mathrm{~ns}$. The delay line compensates the time mismatch between pulses.

Mathematical simulation of the laser showed that under certain conditions, weak feedback for a noise SRS pulse in the cavity results in the generation of a Raman dissipative soliton (RDS) [153]. The RDS has parameters similar to those of the DS. The pulses propagate together in the fiber and form a complex of bound solitons with mutual coherence and high stability, which allows them to be added together, increasing the energy and decreasing the duration of the resulting output pulse. The possibility of generating high-order RDS - multicolor complexes of bound excitons - was also demonstrated numerically.

Model conditions for the appearance of RDSs were later experimentally realized in $[153,154]$. Raman dissipative solitons generated in experiments were compressed to $230 \mathrm{fs}$, which is at the compression level of the main pulse, and experiments on the coherent summation of both pulses were performed. Figure 17 shows the calculated and experimental emission spectra at the cavity output. In the absence of the 

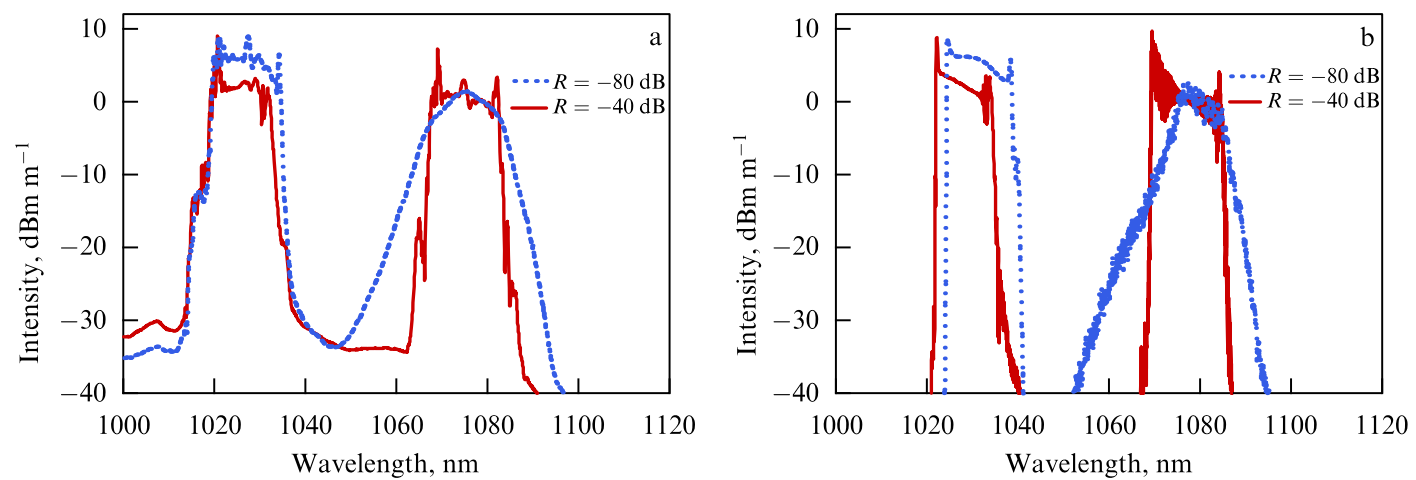

Figure 17. (a) Experimental and (b) calculated spectra of dissipative solitons (DS and RDS) for different feedback coefficients.

feedback or for the feedback coefficient $R<-50 \mathrm{~dB}$, a noise SRS pulse is generated (dashed lines in Fig. 17). As the feedback coefficient increases, the SRS pulse becomes coherent (solid lines in Fig. 17). Coherent pulses have spectra with sharp edges that are typical of strongly chirped dissipative solitons and have linear frequency modulation. It is important that the RDS is a new type of soliton and does not belong to the type of classical Raman solitons which are formed due to the Stokes frequency shift in a fiber with anomalous (negative) dispersion [41], for example, in communication lines [155].

It is important for practical applications that under certain conditions, an RS + RDS complex can be compressed into a single pulse. The dispersion of the DS and RDS was compensated, and then a coherent summation of pulses was performed. After summation, the signal amplitude increases by a factor of four compared to the DS and RDS amplitudes. The resulting pulse duration is $300 \mathrm{fs}$. The shape of the autocorrelation function indicates the presence of amplitude modulation with a peak width of $40 \mathrm{fs}$ and the distance between peaks $75 \mathrm{fs}$, which suggests a high coherence degree for the DS and RDS.

Thus, it was shown that the fundamental restriction caused by the SRS effect can be used to generate a soliton complex with unique properties and promising practical applications. This approach can be developed further, to be used for generating higher-order RDSs, thereby opening the way for creating a source of high-energy (tens of $n J$ ) ultrashort (a few tens of femtoseconds) optical pulses. The potential fields of application of such bound soliton complexes are optical comb spectroscopy, communications lines, and multiphoton fluorescence microscopy.

\subsection{Adiabatic soliton laser}

The temporal and spectral shapes of an optical pulse typically change during its propagation in an optical fiber due to Kerr nonlinearity and group velocity dispersion. Temporal solitons generated in an anomalous dispersion fiber $\left(\beta_{2}<0\right)$ due to the balance between dispersion and nonlinearity do not change their shape during propagation. An important feature of solitons is their stability against small perturbations of the shape and variations in fiber parameters. Thus, as fiber parameters slowly change, the soliton shape is adiabatically adjusted to the varying properties of the medium.

The classical theory of temporal optical solitons is successfully applied in mode-locked fiber lasers for generating spectrally bounded pulses whose parameters can be predicted theoretically $[5,11,65]$. However, the signal the energy in conventional soliton lasers is limited due to generation of scattered radiation (dispersion waves) and passage to multipulse lasing regimes [156]. After periodic amplification in a short part of the active fiber (in each round trip in the cavity), a soliton recovers its shape and loses part of its energy in the form of dispersion waves, which can accumulate up to a significant level, resulting in the breaking of stable single-pulse lasing.

In [157], a new concept of a laser was proposed based on the adiabatic amplification of a signal in a fiber cavity, an adiabatic soliton laser. The adiabatic change in the parameters of a soliton propagating in the cavity allows reducing the constraint on the maximum possible signal energy inherent in conventional soliton lasers.

A schematic of the laser is shown in Fig. 18a. A ring cavity is formed by a standard single-mode fiber (SMF), a $1.5 \mathrm{~m}$ long part of the ytterbium-doped active fiber, and an optical coupler with a $50 \%$ reflection coefficient, an optical filter with a $2 \mathrm{~nm}$ FWHM and the reflection maximum at $1550 \mathrm{~nm}$, and a saturable absorber based on single-wall carbon nanotubes (CNTs). The adiabatic amplification of a soliton in a standard SMF is performed via two-stage SRS amplification. Such a setup of a two-stage SRS amplifier was first proposed in [158] for fiber-optic communication lines. The amplifier setup includes two symmetric pump sources emitting at $1366 \mathrm{~nm}$. A pair of Bragg gratings with the maximum reflection at the wavelength of $1550 \mathrm{~nm}$ of the first Stokes peak form a long laser cavity. The peak power and signal energy at $1550 \mathrm{~nm}$ in this amplifier remain almost constant along the communication line; in other words, the signal propagates without losses.

Unlike the two-stage SRS amplification in communication lines, where the signal power at the fiber input is equal to that at the fiber output (amplification exactly compensates losses in the fiber, $G-\Gamma=0$ ), the signal in the laser cavity is uniformly amplified, and its energy increases after propagation in the fiber $(G-\Gamma>0)$. If the SRS gain is small enough $(G-\Gamma) L_{\mathrm{D}} \ll 1$, the soliton is adiabatically compressed during its propagation, which considerably reduces the influence of dispersion waves.

The influence of distributed Raman amplification on the signal evolution in the cavity was studied in numerical simulations by making comparsons to laser setups with lumped and distributed amplification. The propagation of a signal in a standard single-mode fiber and an erbium-doped fiber is described by the NSE with the amplification 

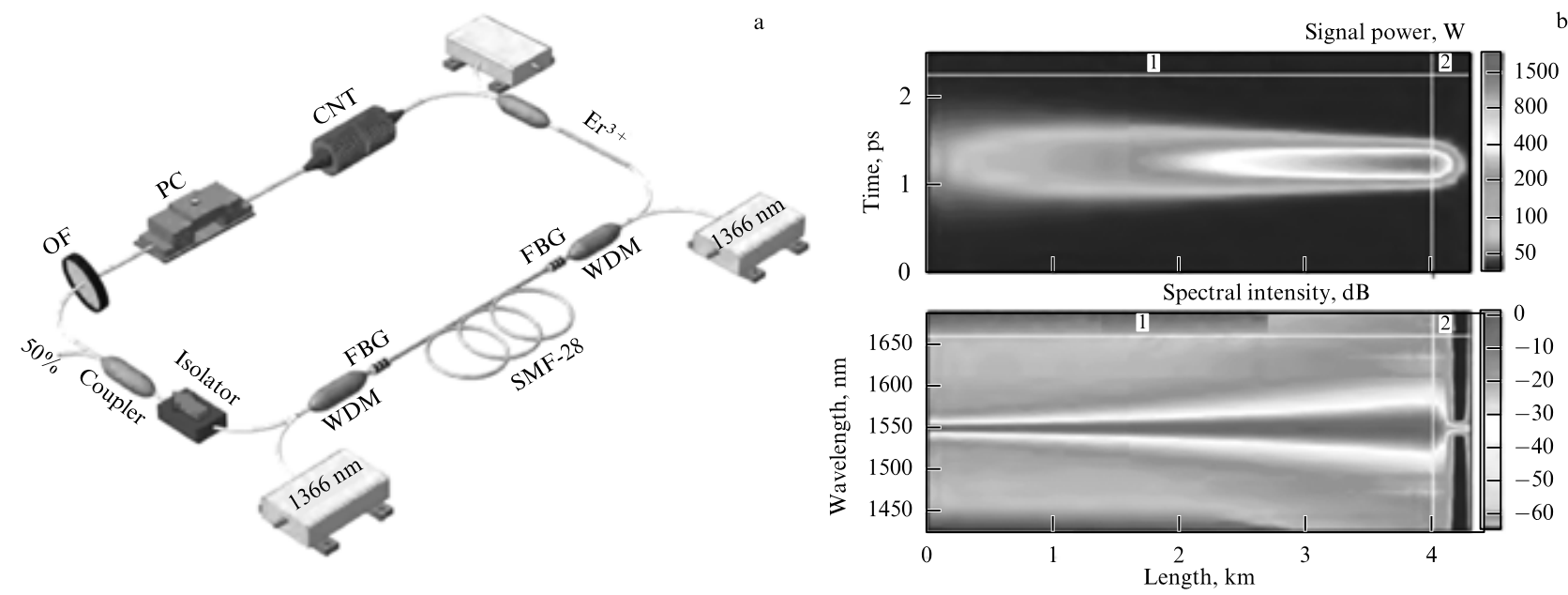

Figure 18. (Color online.) (a) Laser setup. OF — optical filter; PC - polarization controller; WDM - wavelength-division multiplexer; FBG - fiber Bragg grating; CNT — saturable absorber based on single-wall carbon nanotubes; SMF - single-mode fiber. (b) Evolution of the temporal and spectral pulse shape along the cavityr: the long part of an SMF (denoted by ' 1 ') and the short part with mode-locking, filtration, and amplification (denoted by ' 2 ').
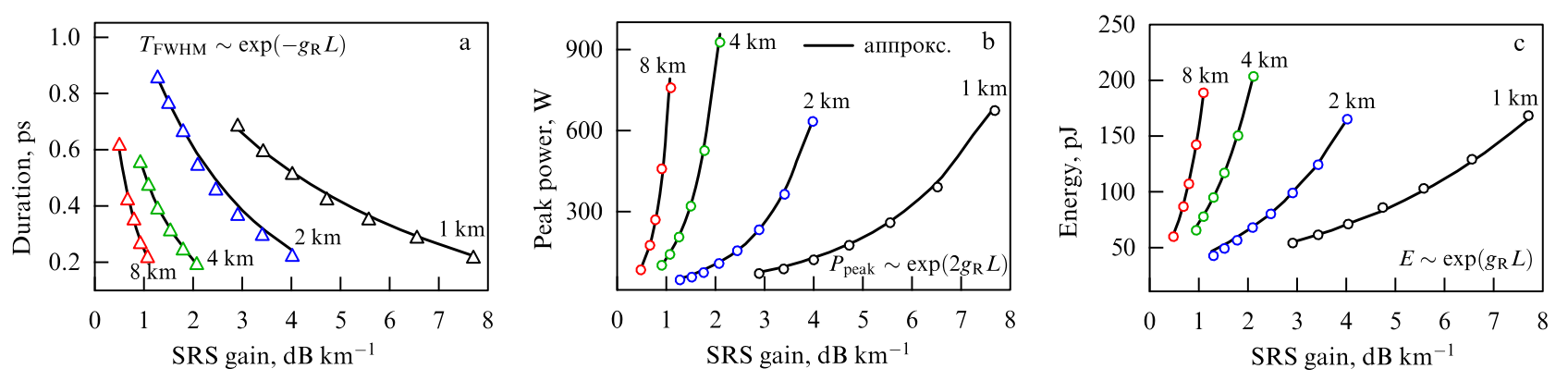

Figure 19. Calculated dependence of the (a) output pulse duration, (b) peak power, and (c) energy on the effective gain (adiabatic soliton amplification regime).

coinciding with (24) if the spectral filtration and selfamplitude modulation and losses are ignored:

$$
\frac{\partial A}{\partial z}=-\frac{\mathrm{i}}{2} \beta_{2} \frac{\partial^{2} A}{\partial t^{2}}+\mathrm{i} \gamma|A|^{2} A+(G-\Gamma) A .
$$

The parameters of an SMF in which a $1550 \mathrm{~nm}$ signal propagates are $\Gamma=0.1 \mathrm{~dB} \mathrm{~km}^{-1}, \beta_{2}=-22.8 \mathrm{ps}^{2} \mathrm{~km}^{-1}$, and $\gamma=1.1 \mathrm{~W}^{-1} \mathrm{~km}^{-1}$.

In the absence of losses in the fiber $(G-\Gamma=0)$, Eqn (52) has an analytic solution in the form of a fundamental soliton [40]:

$$
A(z, t)=\sqrt{P_{\text {sol }}} \operatorname{sech}\left(\frac{t}{\tau_{p}}\right) \exp \left(\frac{\mathrm{i} z}{2 L_{\mathrm{D}}}\right),
$$

where $P_{\text {sol }}=\left|\beta_{2}\right| /\left(\gamma \tau_{p}^{2}\right)$ is the soliton peak power and $T_{\text {sol }}=1.763 \tau_{p}$ is the soliton FWHM duration.

An NSE solution in the form of a bright soliton depends on the dimensionless parameter $N^{2}=L_{\mathrm{D}} / L_{\mathrm{NL}}$, the soliton order, which is a combination of two pulse parameters (peak power and duration) and two fiber parameters (dispersion and nonlinearity). If $N=1$, then the soliton is called the fundamental temporal soliton.

The theoretical hypothesis was verified by simulating the signal propagation in the cavity with the help of NSE (52). The evolution of the spectral and temporal signal shape in the cavity after the establishment of stable lasing is shown in
Fig. 18b. A soliton with a peak power of $60 \mathrm{~W}$ and duration of $1 \mathrm{ps}$ is adiabatically compressed in a $4 \mathrm{~km}$ long standard SMF. Its peak power exponentially increases, $P_{\text {sol }}(z)=$ $P_{\text {sol }}(0) \exp (4(G-\Gamma) z)$, and reaches $1.9 \mathrm{~kW}$, while the pulse duration decreases as $T_{\text {sol }}(z)=T_{\text {sol }}(0) \exp (-2(G-\Gamma) z)$ to $190 \mathrm{fs}$ at $z=4 \mathrm{~km}$. The pulse energy after propagation in the $4 \mathrm{~km}$ fiber is almost six times greater than its input energy. In this case, the soliton order remains equal to unity, which reduces the influence of dispersion waves on the signal.

The soliton perturbation theory describing variations in pulse parameters during adiabatic compression is valid when the condition $(G-\Gamma) z \ll 1$ is satisfied. Stable lasing in the proposed laser setup was observed up to $(G-\Gamma) z \approx 2$. For $(G-\Gamma) z>2$, multipulse regimes appear, which limits the maximum output energy of the laser. Figure 19 shows the dependences of the soliton duration, peak power, and energy at the cavity output on the SRS gain. The soliton energy exponentially increases upon increasing the SRS gain to the level inaccessible for a long-cavity laser with point amplification. Therefore, the signal energy in the fiber laser proposed here exceeds the signal energy achieved in conventional soliton lasers.

\section{Conclusions and an outlook}

Stable dissipative optical solitons (autosolitons) are attractors in corresponding dynamic systems (all trajectories from some vicinity are attracted to their maps in compact regions 
of the phase space) which permits their formation from a wide class of initial distributions of the optical field, including completely random noise distributions. This property makes them attractive for various applications. Dissipative optical solitons have the discrete spectra of main parameters such as pulse energy and width. This property of dissipative solitons results in the suppression of the drift of soliton parameters under the action of fluctuations, which provides their stability against the influence of optical and other noises. Dissipative solitons in lasers allow a high energy to be localized in short pulses without separating of a soliton into several pulses and without the appearance of additional radiation. The nonlinear physics of the generation of dissipative optical solitons in lasers is of interest not only for applications but also for fundamental studies. Mathematical models describing dissipative optical solitons in lasers can be used in many physical and even biological problems. The theory of dissipative solitons in fiber lasers provides wide possibilities for generating and controlling such pulses, but, on the other hand, requires knowledge of the soliton theory and nonlinear physics. The development of effective soliton lasers requires combined efforts of theorists and engineers.

The necessary condition for the stability of dissipative solitons in lasers is the stability of the nonlasing regime. This means the bistability (multistability) of the system because, along with this regime, one of the continuous or pulsed lasing regimes can also be excited. The hard type of excitation of such solitons ensures their high stability due to efficient noise suppression. In fiber lasers, aside from the 'conservative' nonresonance nonlinearity of the refractive index, the nonresonance nonlinearity of amplification and absorption and their inertia are very important. Although dissipative laser solitons also exist in the limit of the inertialess resonance nonlinearity, the presence of nonlinearity relaxation substantially changes their properties, giving rise to new types of stable localized pulses. It seems that the physics of ultrashort dissipative solitons based on self-induced transparency'attosolitons' in fiber lasers with saturable absorption in the coherent self-mode-locking regime - is far from being completely investigated. Of course, the examples of dissipative solitons considered here cannot fully encompass this huge and rapidly developing field. We hope that our review gives a picture of some promising studies and recent results in the field of applications of dissipative optical solitons in fiber lasers and will attract attention to this interesting field of science and technology.

\section{Acknowledgments}

The work by M P Fedoruk was supported by the Russian Scientific Foundation (project no. 14-21-00110). The work by S K Turitsyn was supported by the Ministry of Education and Science of the Russian Federation (project no. 14.B25.31.0003). The work by $\mathrm{N} N$ Rozanov was supported by the Russian Foundation for Basic Research (grant no. 14-02-90026_Bel_a). S K Turitsyn, B N Rozanov, and S V Fedorov acknowledge the support of the Leading Universities of the Russian Federation (subsidy 074-U01).

\section{References}

1. Zabusky N J, Kruskal M D Phys. Rev. Lett. 15240 (1965)

2. Scott A Nonlinear Science. Emergence and Dynamics of Coherent Structures (Oxford: Oxford Univ. Press, 1999)
3. Novikov S et al. Theory of Solitons. The Inverse Scattering Method (New York: Consultants Bureau, 1984); Translated from Russian: Zakharov V E et al. Teoriya Solitonov: Metod Obratnoi Zadachi (Moscow: Nauka, 1980)

4. Newell A C Solitons in Mathematics and Physics (Philadelphia, Pa.: SIAM, 1985)

5. Mollenauer L F, Gordon J P Solitons in Optical Fibers. Fundamentals and Applications (Amsterdam: Elsevier, Academic Press, 2006)

6. Hasegawa A, Kodama Y Solitons in Optical Communications (Oxford: Clarendon Press, 1995)

7. Iannone E et al. Nonlinear Optical Communication Networks (New York: Wiley, 1998)

8. Taylor J R (Ed.) Optical Solitons: Theory and Experiment (Cambridge Studies in Modern Optics, Vol. 10) (Cambridge: Cambridge Univ. Press, 1992)

9. Akhmediev N, Ankiewicz A, in Dissipative Solitions (Lecture Notes in Physics, Vol. 661, Eds. N Akhmediev, A Ankiewicz) (Berlin: Springer, 2005) p. 1; Translated into Russian: Dissipativnye Solitony (Eds N Akhmediev, A Ankiewicz) (Moscow: Nauka, 2008)

10. Akhmediev N, Ankiewicz A, in Dissipative Solitons. From Optics to Biology and Medicine (Lecture Notes in Physics, Vol. 751, Eds N Akhmediev, A Ankiewicz) (Berlin: Springer, 2008) p. 1

11. Kivshar Y S, Agrawal G P Optical Solitons: From Fibers to Photonic Crystals (Amsterdam: Academic Press, 2003); Translated into Russian: Opticheskie Solitony. Ot Volokonnykh Svetovodov do Fotonnykh Kristallov (Moscow: Fizmatlit, 2005)

12. Ablowitz M J, Segur H Solitons and the Inverse Scattering Transform (Philadelphia: SIAM, 1981); Translated into Russian: Solitony $i$ Metod Obratnoi Zadachi (Moscow: Mir, 1987)

13. Calogero F, Degasperis A Spectral Transform and Solitons Vol. 1 (Amsterdam: North-Holland, 1982)

14. Ablowitz M J, Clarkson P A Solitons, Nonlinear Evolution Equations and Inverse Scattering (Cambridge: Cambridge Univ. Press, 1991)

15. Lonngren K, Scott A (Eds) Solitons in Action. Proc. of a Workshop, Redstone Arsenal, October 26-27, 1977 (New York: Academic Press, 1978)

16. Dodd R K et al. Solitons and Nonlinear Wave Equations (London: Academic Press, 1982); Translated into Russian: Solitony i Nelineinye Volnovye Uravneniya (Moscow: Mir, 1988)

17. Trullinger S E, Zakharov V E, Pokrovsky V L (Eds) Solitons (Modern Problems in Condensed Matter Sciences, Vol. 17) (Amsterdam: North-Holland, 1986)

18. Kerner B S, Osipov V V Autosolitons: a New Approach to Problems of Self-Organization and Turbulence (Dordrecht: Kluwer Acad. Publ., 1994); Translated from Russian: Avtosolitony. Lokalizovannye Sil'no-neravnovesnye Oblasti $v$ Odnorodnykh Dissipativnykh Sistemakh (Moscow: Nauka, 1991)

19. Remoissenet M Waves Called Solitons: Concepts and Experiments (Berlin: Springer-Verlag, 1994)

20. Malomed B A Soliton Management in Periodic Systems (New York: Springer, 2006); Translated into Russian: Kontrol' Solitonov v Periodicheskikh Sredakh (Moscow: Fizmatlit, 2009)

21. Infeld E, Rowlands G Nonlinear Waves, Solitons, and Chaos (Cambridge: Cambridge Univ. Press, 1990); Translated into Russian: Nelineinye Volny, Solitony i Khaos (Moscow: Fizmatlit, 2005)

22. Rajaraman R Solitons and Instantons. An Introduction to Solitons and Instantons in Quantum Field Theory (Amsterdam: North-Holland, 1982)

23. Kossevich A M The Crystal Lattice: Phonons, Solitons, Dislocations (Berlin: Wiley-VCH, 1999)

24. Manton N, Sutcliffe P Topological Solitons (Cambridge: Cambridge Univ. Press, 2004)

25. Ginzburg V L, Landau L D, in Landau L D Collected Papers (Oxford: Pergamon Press, 1965) p. 546; Zh. Eksp. Teor. Fiz. 201064 (1950)

26. Newell A C, Whitehead J A J. Fluid Mech. 38279 (1969)

27. Eckhaus W Studies in Non-Linear Stability Theory (Springer Tracts in Natural Philosophy, Vol. 6) (Berlin: Springer, 1965)

28. Haus H A J. Appl. Phys. 463049 (1975)

29. Newell A C Nonlinear Wave Motion (Lectures in Applied Mathematics, Vol. 15) (Providence, RI: American Mathematical Society, 1974) 
30. Kuramoto Y Chemical Oscillations, Waves, and Turbulence (Berlin: Springer-Verlag, 1984)

31. Bogolubov N J. Phys. USSR 1123 (1947); http://ufn.ru/pdf/ jphysussr/1947/11_1/3jphysussr 19471101.pdf

32. Gross E P Nuovo Cimento 20454 (1961)

33. Pitaevskii L P Sov. Phys. JETP 13451 (1961); Zh. Eksp Teor. Fiz. 40 646 (1961)

34. Chiao R Y, Garmire E, Townes C H Phys. Rev. Lett. 13479 (1964)

35. Kelley P L Phys. Rev. Lett. 151005 (1965)

36. Talanov V I JETP Lett. 2138 (1965); Pis'ma Zh. Eksp Teor. Fiz. 2 218 (1965)

37. Zakharov V E Sov. Phys. JETP 26994 (1968); Zh. Eksp. Teor. Fiz. 531735 (1967)

38. Zakharov V J. Appl. Mech. Tech. Phys. 9190 (1968); Zh. Prikl. Mekh. Teor. Fiz. 9 (2) 86 (1968)

39. Kuznetsov E A, Mikhailov A V, Shimokhin I A Physica D 87201 (1995)

40. Zakharov V E, Shabat A B Sov. Phys. JETP 3462 (1972); Zh. Eksp. Teor. Fiz. 61118 (1971)

41. Agrawal G P Nonlinear Fiber Optics 3rd ed. (San Diego: Academic Press, 2001)

42. Haus H A, Fujimoto J G, Ippen E P J. Opt. Soc. Am. B 82068 (1991)

43. Haus H A, Mecozzi A IEEE J. Quantum Electron. 29983 (1993)

44. Rozanov N N, Fedorov S V Opt. Spectrosc. 72782 (1992); Opt. Spektrosk. 721394 (1992)

45. Rozanov N N, Fedorov S V Opt. Spectrosc. 84767 (1998); Opt. Spektrosk. 84849 (1998)

46. Rozanov N N, Fedorov S V Opt. Spectrosc. 72782 (1992); Opt. Spectrosk. 88869 (2000)

47. Fedorov S V et al. Phys. Rev. E 615814 (2000)

48. Grelu P, Akhmediev N Nature Photon. 684 (2012)

49. Khanin Ya I Kvanovaya Radifizika (Quantum Radiophysics) Vol. 2 Dinamika Kvantovykh Generatorov (Dynamics of Lasers) (Moscow: Sov. Radio, 1975)

50. Kozlov V V Phys. Rev. A 561607 (1997)

51. Turitsyn S K et al. Opt. Express 198394 (2011)

52. Kobtsev S, Kukarin S, Fedotov Y Opt. Express 1621936 (2008)

53. Kobtsev S et al., CLEO/Europe CJ8.4 (2009)

54. Nyushkov B N et al. Laser Phys. Lett. 7661 (2010)

55. Lim H, Ilday F Ö, Wise F W Opt. Lett. 28660 (2003)

56. Lefrançois S et al. Opt. Lett. 351569 (2010)

57. Tang D Y et al. Phys. Rev. A 72043816 (2005)

58. Komarov A, Leblond H, Sanchez F Phys. Rev. E 72 025604(R) (2005)

59. Kutz J N SIAM Rev. 48629 (2006)

60. Aranson I S, Kramer L Rev. Mod. Phys. 7499 (2002)

61. Haus H A, Fujimoto J G, Ippen E P IEEE J. Quantum Electon. 28 2086 (1992)

62. Haus H A IEEE J. Sel. Top. Quantum Electron. 61173 (2000)

63. Lega J, Moloney J V, Newell A C Phys. Rev. Lett. 732978 (1994)

64. Podivilov E, Kalashnikov V L JETP Lett. 82467 (2005); Pis'ma Zh. Eksp. Teor. Fiz. 82524 (2005)

65. Turitsyn S K, Bale B G, Fedoruk M P Phys. Rep. 521135 (2012)

66. Komarov K P Opt. Spectrosc. 60231 (1986); Opt. Spektrosk. 60379 (1986)

67. Komarov K P, Kuch'yanov A S, Ugozhayev V D Opt. Commun. 57 279 (1986)

68. Lazaridis P, Debarge G, Gallion P Opt. Lett. 201160 (1995)

69. Proctor B, Westwig E, Wise F Opt. Lett. 181654 (1993)

70. Kapitula T, Kutz J N, Sandstede B J. Opt. Soc. Am. B 19740 (2002)

71. Soto-Crespo J M, Akhmediev N N, Afanasjev V V J. Opt. Soc. Am. $B 131439$ (1996)

72. Soto-Crespo J M et al. Phys. Rev. E55 4783 (1997)

73. Akhmediev N et al. Opt. Fiber Technol. 11209 (2005)

74. Biondini G Nonlinearity 212849 (2008)

75. Fuerbach A et al. Laser Part. Beams 23113 (2005)

76. Chong A et al. Opt. Express 1410095 (2006)

77. Renninger W H, Chong A, Wise F W Phys. Rev. A 77023814 (2008)

78. Bale B G et al. J. Opt. Soc. Am. B 251763 (2008)

79. Renninger W H, Chong A, Wise F W J. Opt. Soc. Am. B 271978 (2010)

80. Kalashnikov V L et al. Appl. Phys. B 83503 (2006)

81. Kalashnikov V L Phys. Rev. E 80046606 (2009)
82. Kharenko D S et al. J. Opt. Soc. Am. B 282314 (2011)

83. Kalashnikov V L, Apolonski A Phys. Rev. A 79043829 (2009)

84. Anderson D et al. J. Opt. Soc. Am. B 101185 (1993)

85. Fermann M E et al. Phys. Rev. Lett. 846010 (2000)

86. Akhmediev N N, Eleonskii V M, Kulagin N E Theor. Math. Phys. 72809 (1987); Teor. Mat. Fiz. 72183 (1987)

87. Soto-Crespo J M, Akhmediev N, Town G J. Opt. Soc. Am. B 19234 (2002)

88. Shtyrina O V et al. IEEE Photon. J. 71501207 (2015)

89. Struwe M Variational Methods. Applications to Nonlinear Partial Differential Equations and Hamiltonian Systems 3rd ed. (Berlin: Springer, 2000)

90. Anderson D Phys. Rev. A 273135 (1983)

91. Malomed B, in Progress in Optics Vol. 43 (Ed E Wolf) (Amsterdam: North-Holland, 2002) Ch. 2

92. Turitsyn S K, Shapiro E G Opt. Fiber Technol. 4151 (1998)

93. Gabitov I, Turitsyn S K JETP Lett. 63861 (1996); Pis'ma Zh. Eksp. Teor. Fiz. 63814 (1996)

94. Turitsyn S K, Mezentsev V K JETP Lett. 67640 (1998); Pis'ma Zh. Eksp. Teor. Fiz. 67616 (1998)

95. Turitsyn S K et al. Comptes Rendus Phys. 4145 (2003)

96. Vlasov S N, Petrishchev V A, Talanov V I Radiophys. Quantum Electron. 141062 (1971); Izv. Vyssh. Uchebn. Zaved. Radiofiz. 14 1353 (1971)

97. Berkshire F H, Gibbon J D Stud. Appl. Math. 69229 (1983)

98. Marcuse D IEEE J. Light. Tech. 1017 (1992)

99. Bélanger P-A, Bélanger N Opt. Commun. 11756 (1995)

100. Shapiro E G, Turitsyn S K Opt. Lett. 221544 (1997)

101. Turitsyn S K, Schäfer T, Mezentsev V K Opt. Lett. 231351 (1998)

102. Turitsyn $\mathrm{S}$ K et al. Phys. Rev. E 59 3843(R) (1999)

103. Turitsyn S K Phys. Rev. E 58 R 1256 (1998)

104. Schreiber T et al. Opt. Express 158252 (2007)

105. Shtyrina O et al. J. Opt. Soc. Am. B 26346 (2009)

106. Bale B G et al. Phys. Rev. A 81033828 (2010)

107. Tian X et al. Opt. Express 177222 (2009)

108. Bale B G, Okhotnikov O G, Turitsyn S K, in Fiber Lasers (Ed. O G Okhotnikov) (Weinheim: Wiley-VCH, 2012) p. 135

109. Namiki S et al. J. Opt. Soc. Am. B 142099 (1997)

110. Gabitov I R, Turitsyn S K Opt. Lett. 21327 (1996)

111. Shapiro E G, Turitsyn S K Phys. Rev. E 56 R4951 (1997)

112. Wise F W, Chong A, Renninger W H Laser Photon. Rev. 258 (2008)

113. Turitsyn S K, Schaefer T, Mezentsev V K Phys. Rev. E 58 R5264 (1998)

114. Shtyrina O V, Yarutkina I A, Fedoruk M P Opt. Express 2231814 (2014)

115. Vanin E A et al. Phys. Rev. A 492806 (1994)

116. Khanin Ya I Fundamentals of Laser Dynamics (Cambridge: Cambridge Intern. Sci. Publ., 2006)

117. Morgner U et al. Opt. Lett. 24411 (1999)

118. Sutter D H et al. Opt. Lett. 24631 (1999)

119. Ell R et al. Opt. Lett. 26373 (2001)

120. McCall S L, Hahn E L Phys. Rev. 183457 (1969)

121. Allen L, Eberly J H Optical Resonance and Two-Level Atoms (New York: Dover, 1987); Translated into Russian: Opticheskii Rezonans $i$ Dvukhurovnevye Atomy (Moscow: Mir, 1978)

122. Arkhipov M V et al. JETP Lett. 101232 (2015); Pis'ma Zh. Eksp. Teor. Fiz. 101250 (2015)

123. Vysotina N V et al. Opt. Spectrosc. 101736 (2006); Opt. Spektrosk. 101784 (2006)

124. Vysotina N V, Rozanov N N, Semenov V E JETP Lett. 83279 (2006); Pis'ma Zh. Eksp. Teor. Fiz. 83337 (2006)

125. Rosanov N N, Semenov V E, Vyssotina N V Laser Phys. 171311 (2007)

126. Rosanov N N, Semenov V E, Vysotina N V Quantum Electron. 38 137 (2008); Kvantovaya Elektron. 38137 (2008)

127. Vysotina N V, Rosanov N N, Semenov V E Opt. Spectrosc. 106713 (2009); Opt. Spektrosk. 106793 (2009)

128. Rozanov N N Dissipativnye Opticheskie Solitony: ot Mikro- $k$ Nano$i$ Atto (Dissipative Optical Solitons: from Micro to Nano and Atto) (Moscow: Fizmatlit, 2011)

129. Kozlov V V, Rosanov N N, Wabnitz S Phys. Rev. A 84053810 (2011) 
130. Kozlov V V, Rosanov N N Opt. Spectrosc. 114798 (2013); Opt. Spektrosk. 114870 (2013)

131. Kozlov V V, Rosanov N N Phys. Rev. A 87043836 (2013)

132. Rosanov N N, Kozlov V V, Wabnitz S Phys. Rev. A 81043815 (2010)

133. Kozlov V V, Rosanov N N Opt. Spectrosc. 116956 (2014); Opt. Spektrosk. 1161033 (2014)

134. Siegman A E Lasers (Mill Valley, Calif.: Univ. Science Books, 1986)

135. Turitsyn $\mathrm{S}$ et al., in 36th European Conf. and Exhibition on Optical Communication (ECOC) (Piscataway, NJ: IEEE, 2010) Tu.5.D.1

136. Smirnov S V, Kobtsev S M, Kukarin S V, Turitsyn S K, in Laser Systems for Applications (Ed. K Jakubczak) (Rijeka, Croatia: InTech, 2011) Ch. 3

137. Kobtsev S et al. Proc. SPIE 7580758028 (2010)

138. Kobtsev S M, Smirnov S V Laser Phys. 21272 (2011)

139. Li N et al. Appl. Opt. 513726 (2012)

140. Oktem B, Ülgüdür C, Ilday F Ö Nature Photon. 4307 (2010)

141. Chernykh A I, Turitsyn S K Opt. Lett. 20398 (1995)

142. Tang D Y, Zhao L M, Zhao B Opt. Express 132289 (2005)

143. Yarutkina I A et al. Opt. Express 2112942 (2013)

144. Okhotnikov O G et al. Opt. Lett. 281522 (2003)

145. Kelley S M J Electron. Lett. 28806 (1992)

146. Haus H A et al. IEEE J. Quantum Electron. 31591 (1995)

147. Tamura K et al. Appl. Phys. Lett. 64149 (1994)

148. Grigoryan V S, Muradyan T S J. Opt. Soc. Am. B 81757 (1991)

149. Kharenko D S et al. Laser Phys. Lett. 9662 (2012)

150. Renninger W H, Wise F W, in Fiber Lasers (Ed. O G Okhotnikov) (Weinheim:Wiley-VCH, 2012) p. 97

151. Kharenko D S et al. Opt. Lett. 374104 (2012)

152. Bednyakova A E et al. Opt. Express 2120556 (2013)

153. Babin S A et al. Nature Commun. 54653 (2014)

154. Kharenko D S et al. Opt. Express 231857 (2015)

155. Kumar S, Hasegawa A Opt. Lett. 201856 (1995)

156. Mollenauer L F, Stolen R H Opt. Lett. 913 (1984)

157. Bednyakova A, Turitsyn S K Phys. Rev. Lett. 114113901 (2015)

158. Ania-Castañón J D et al. Phys. Rev. Lett. 96023902 (2006) 